\title{
A global QP-free algorithm for mathematical programs with complementarity constraints
}

Jian Ling Li $i^{* *}$ and Qi Zhang

Correspondence:

jianlingli@126.com

${ }^{1}$ College of Mathematics and

Information Science, Guangxi

University, Nanning, China

\section{Springer}

\begin{abstract}
In this paper, a primal-dual interior point QP-free algorithm for mathematical programs with complementarity constraints is presented. Firstly, based on Fischer-Burmeister function and smoothing techniques, the investigated problem is approximated by a smooth nonlinear constrained optimization problem. Secondly, combining with an effective penalty function technique and working set, a QP-free algorithm is proposed to solve the smooth constrained optimization problem. At each iteration, only two reduced linear equations with the same coefficient matrix are solved to obtain the search direction. Under some mild conditions, the proposed algorithm possesses global convergence. Finally, some numerical results are reported.
\end{abstract}

Keywords: Complementarity constraints; Working set; QP-free algorithm; Global convergence

\section{Introduction}

In this paper, we discuss the following mathematical programming problem with complementarity constraints (MPCC for short):

$$
\begin{array}{ll}
\min & f(x, y) \\
\text { s.t. } & g(x, y) \leq 0, \\
& 0 \leq F(x, y) \perp y \geq 0,
\end{array}
$$

where $f: \mathbb{R}^{n+m} \rightarrow \mathbb{R}, g=\left(g_{1}, \ldots, g_{m_{g}}\right)^{\mathrm{T}}: \mathbb{R}^{n+m} \rightarrow \mathbb{R}^{m_{g}}, F=\left(F_{1}, \ldots, F_{m}\right)^{\mathrm{T}}: \mathbb{R}^{n+m} \rightarrow \mathbb{R}^{m}$ are continuously differentiable functions. " $F(x, y) \perp y$ " means that the vectors $F(x, y)$ and $y$ are perpendicular to each other.

MPCC (1) has a broad of applications in real world, such as engineering design, traffic transportation, game theory and so on. A detailed overview of MPCC applications can be found in [1] and the monographs [2-4].

Since MPCC (1) is a nonconvex optimization problem and the standard MangasarianFromovitz constraint qualification (MFCQ) is violated at any feasible point, the welldeveloped algorithms for the standard nonlinear programs (for example, [5-18]) typically have severe difficulties if they are directly used to solve the MPCC (1). Hence, MPCCtailed algorithms are desired.

(c) The Author(s) 2020. This article is licensed under a Creative Commons Attribution 4.0 International License, which permits use, sharing, adaptation, distribution and reproduction in any medium or format, as long as you give appropriate credit to the original author(s) and the source, provide a link to the Creative Commons licence, and indicate if changes were made. The images or other third party material in this article are included in the article's Creative Commons licence, unless indicated otherwise in a credit line to the material. If material is not included in the article's Creative Commons licence and your intended use is not permitted by statutory regulation or exceeds the permitted use, you will need to obtain permission directly from the copyright holder. To view a copy of this licence, visit http://creativecommons.org/licenses/by/4.0/. 
It is well known that the QP-free method is one of the efficient methods for nonlinear programming (see [14, 15, 19-25] ). The nice properties of the QP-free method are as follows: (a) The search directions are determined only by solving systems of linear equations rather than solving QP-subproblems. As a consequence, the computational cost is decreased greatly. (b) Compared with the SQP method, the size of the investigated problem solved by QP-free method is larger. It is worth mentioning that the primal-dual interior point QP-free algorithm in [21] has an improvement, that is, the strict restriction that the Lagrangian Hessian estimate is uniformly positive is relaxed. It is noticed that the active set identifying technique is incorporated into the QP-free algorithms in [22, 23]. Consequently, the computational cost is further decreased and the numerical performance in [22] is encouraging.

Although there are many OP-free algorithms for nonlinear programming, to the best of our knowledge, there are few MPCC-tailed QP-free algorithms for the MPCC (1). Motivated by the ideas of the algorithms in [21, 24-27] and combining with smoothing techniques, we propose a primal-dual interior point QP-free algorithm for the MPCC (1). The proposed algorithm possesses the following nice properties:

(a) The technique of active set is incorporated into the algorithm. As a consequence, the size of SLEs becomes smaller and the computational cost is decreased.

(b) At each iteration, the search direction is obtained by solving two SLEs with the same coefficient matrix, which further decreases the computational cost.

(c) The uniformly positive definiteness on the Lagrangian Hessian estimate $H_{k}$ is relaxed.

(d) The algorithm possesses global convergence without assuming that the stationary points are isolated.

\section{Preliminaries and reformulation}

In this section, for completeness, we first restate some definitions and results about the MPCC (1), then deduce an approximation problem of the MPCC (1) by FischerBurmeister function and smoothing techniques.

Denoted by $X_{0}$ the feasible set of the MPCC (1), and denoted by $I^{\mathcal{C}}=\{1,2, \ldots, m\}$ and $I^{g}=\left\{1,2, \ldots, m_{g}\right\}$ are the index sets of the complementarity constraints and the inequality constraints, respectively.

Definition 1 ([2]) Given $\left(x^{*}, y^{*}\right) \in X_{0}$, if

$$
\left(y_{i}^{*}, F_{i}\left(x^{*}, y^{*}\right)\right) \neq(0,0), \quad \forall i \in I^{\mathcal{C}}
$$

then we call that the lower-level strict complementarity (LLSC) is satisfied at $\left(x^{*}, y^{*}\right)$.

Definition 2 ([2]) A point $\left(x^{*}, y^{*}\right) \in X_{0}$ is said to be a KKT stationary point of the MPCC (1) if there exists a KKT multiplier vector $\left(\lambda^{*}, \omega^{*}, \mu^{*}\right) \in \mathbb{R}^{m+m+m_{g}}$ such that

$$
\begin{aligned}
& \nabla f\left(x^{*}, y^{*}\right)+\nabla g\left(x^{*}, y^{*}\right) \lambda^{*}-\nabla F\left(x^{*}, y^{*}\right) \omega^{*}-\left(\begin{array}{c}
0_{n \times m} \\
E_{m}
\end{array}\right) \mu^{*}=0, \\
& 0 \leq-g\left(x^{*}, y^{*}\right) \perp \lambda^{*} \geq 0 ; \quad F\left(x^{*}, y^{*}\right) \geq 0, \quad y^{*} \geq 0, \quad F_{i}\left(x^{*}, y^{*}\right) y_{i}^{*}=0 ; \\
& \omega_{i}^{*}=0, \quad \text { if } F_{i}\left(x^{*}, y^{*}\right)>0 ; \quad \mu_{i}^{*}=0, \quad \text { if } y_{i}^{*}>0 ;
\end{aligned}
$$




$$
\omega_{i}^{*} \geq 0, \quad \mu_{i}^{*} \geq 0, \quad \text { if } F_{i}\left(x^{*}, y^{*}\right)=y_{i}^{*}=0,
$$

where $E_{m} \in \mathbb{R}^{m \times m}$ is an $m$ th-order identity matrix. The vector $\left(\left(x^{*}, y^{*}\right), \lambda^{*}, \omega^{*}, \mu^{*}\right)$ is said to be a KKT stationary pair of the MPCC (1).

Proposition 1 ([2]) Suppose that $\left(x^{*}, y^{*}\right) \in X_{0}$ satisfies LLSC, then $\left(\left(x^{*}, y^{*}\right), \lambda^{*}, \omega^{*}, \mu^{*}\right)$ is a KKT stationary pair of the MPCC (1) if and only if there exists a vector $\iota^{*}=\left(\iota_{i}^{*}, i \in I^{\mathcal{C}}\right) \in \mathbb{R}^{m}$, such that

$$
\begin{aligned}
& \left(\begin{array}{c}
\nabla_{x} f\left(x^{*}, y^{*}\right) \\
\nabla_{y} f\left(x^{*}, y^{*}\right) \\
0_{m \times 1}
\end{array}\right)+\left(\begin{array}{c}
\nabla_{x} g\left(x^{*}, y^{*}\right) \\
\nabla_{y} g\left(x^{*}, y^{*}\right) \\
0_{m \times l}
\end{array}\right) \lambda^{*}-\left(\begin{array}{c}
\nabla_{x} F\left(x^{*}, y^{*}\right) \\
\nabla_{y} F\left(x^{*}, y^{*}\right) \\
-E_{m}
\end{array}\right) \omega^{*}-\left(\begin{array}{c}
0_{n \times m} \\
V^{*} \\
Y^{*}
\end{array}\right) \iota^{*}=0, \\
& 0 \leq-g\left(x^{*}, y^{*}\right) \perp \lambda^{*} \geq 0, \quad 0 \leq F\left(x^{*}, y^{*}\right) \perp y^{*} \geq 0, \\
& \iota_{i}^{*}= \begin{cases}\mu_{i}^{*} / F_{i}\left(x^{*}, y^{*}\right), & \text { if } F_{i}\left(x^{*}, y^{*}\right)>0, \\
\omega_{i}^{*} / y_{i}^{*}, & \text { if } y_{i}^{*}>0,\end{cases}
\end{aligned}
$$

where $V^{*}=\operatorname{diag}\left(F_{i}\left(x^{*}, y^{*}\right), i \in I^{\mathcal{C}}\right), Y^{*}=\operatorname{diag}\left(y_{i}^{*}, i \in I^{\mathcal{C}}\right)$.

It is well known that the Fischer-Burmeister function is a complementarity function, which is defined by

$$
\psi(a, b)=a+b-\sqrt{a^{2}+b^{2}} .
$$

Obviously, $\psi$ satisfies the following basic property:

$$
\psi(a, b)=0 \quad \Longleftrightarrow \quad a \geq 0, b \geq 0, a b=0 .
$$

And $\psi$ is continuous differentiable in $\left\{(a, b) \in \mathbb{R}^{2} \mid(a, b) \neq(0,0)\right\}$, namely

$$
\psi_{a}^{\prime}(a, b)=1-\frac{a}{\sqrt{a^{2}+b^{2}}} ; \quad \psi_{b}^{\prime}(a, b)=1-\frac{b}{\sqrt{a^{2}+b^{2}}}, \quad(a, b) \neq(0,0)
$$

Let $v=F(x, y)$, according to the property (3), the MPCC (1) is equivalently transformed into the following nonsmooth nonlinear optimization problem:

$$
\begin{array}{ll}
\min & f(x, y) \\
\text { s.t. } & v-F(x, y)=0, \\
& \Psi(y, v)=0, \\
& g(x, y) \leq 0
\end{array}
$$

where $\Psi(y, v)=\left(\psi\left(y_{i}, v_{i}\right), i \in I^{\mathcal{C}}\right)$.

Obviously, the function $\psi$ is not differentiable at the point $(0,0)$. Borrowing ideas from [28], we define the functions as follows:

$$
\psi_{\varepsilon}\left(y_{i}, v_{i}\right)= \begin{cases}\psi\left(y_{i}, v_{i}\right), & i \in I^{\mathcal{C}} \backslash I^{\mathcal{C}}(y, v, \varepsilon) \\ \frac{2 \varepsilon-y_{i}}{2 \varepsilon} y_{i}+\frac{2 \varepsilon-v_{i}}{2 \varepsilon} v_{i}-\frac{\varepsilon}{2}, & i \in I^{\mathcal{C}}(y, v, \varepsilon)\end{cases}
$$




$$
\bar{\psi}_{\varepsilon}\left(y_{i}, v_{i}\right)= \begin{cases}0, & i \in I^{\mathcal{C}} \backslash I^{\mathcal{C}}(y, v, \varepsilon), \\ \frac{\left(\sqrt{y_{i}^{2}+v_{i}^{2}}-\varepsilon\right)^{2}}{2 \varepsilon}, & i \in I^{\mathcal{C}}(y, v, \varepsilon),\end{cases}
$$

where the index $\operatorname{set} I^{\mathcal{C}}(y, v, \varepsilon)=\left\{i \in I^{\mathcal{C}} \mid \sqrt{y_{i}^{2}+v_{i}^{2}}<\varepsilon, \varepsilon>0\right\}$. Moreover, define

$$
\Psi_{\varepsilon}(y, v)=\left(\psi_{\varepsilon}\left(y_{i}, v_{i}\right), i \in I^{\mathcal{C}}\right), \quad \bar{\Psi}_{\varepsilon}(y, v)=\left(\bar{\psi}_{\varepsilon}\left(y_{i}, v_{i}\right), i \in I^{\mathcal{C}}\right),
$$

so we have

$$
\Psi(y, v)=\Psi_{\varepsilon}(y, v)+\bar{\Psi}_{\varepsilon}(y, v)
$$

For any $\varepsilon>0$, the function $\psi_{\varepsilon}\left(y_{i}, v_{i}\right)$ is differentiable, and it follows that

$$
\begin{aligned}
& \psi_{\varepsilon, y_{i}}^{\prime}\left(y_{i}, v_{i}\right)=\frac{\partial \psi_{\varepsilon}\left(y_{i}, v_{i}\right)}{\partial y_{i}}= \begin{cases}1-\frac{y_{i}}{\sqrt{y_{i}^{2}+v_{i}^{2}},} & i \in I^{\mathcal{C}} \backslash I^{\mathcal{C}}(y, v, \varepsilon), \\
1-\frac{y_{i}}{\varepsilon}, & i \in I^{\mathcal{C}}(y, \nu, \varepsilon),\end{cases} \\
& \psi_{\varepsilon, v_{i}}^{\prime}\left(y_{i}, v_{i}\right)=\frac{\partial \psi_{\varepsilon}\left(y_{i}, v_{i}\right)}{\partial v_{i}}= \begin{cases}1-\frac{v_{i}}{\sqrt{y_{i}^{2}+v_{i}^{2}},} & i \in I^{\mathcal{C}} \backslash I^{\mathcal{C}}(y, v, \varepsilon), \\
1-\frac{v_{i}}{\varepsilon}, & i \in I^{\mathcal{C}}(y, \nu, \varepsilon) .\end{cases}
\end{aligned}
$$

So the smooth problem

$$
\begin{array}{ll}
\min & f(x, y) \\
\text { s.t. } & v-F(x, y)=0, \\
& \Psi_{\varepsilon}(y, v)=0, \\
& g(x, y) \leq 0
\end{array}
$$

will be used as an approximation of the MPCC (1). Obviously, if $I^{\mathcal{C}}\left(y^{*}, v^{*}, \varepsilon\right)=\emptyset$, then the problem (6) is equivalent with the problem (4). Under some mild conditions, $I^{\mathcal{C}}\left(y^{*}, v^{*}, \varepsilon\right)=$ $\emptyset$ is guaranteed, where $\left(x^{*}, y^{*}, v^{*}\right)$ is an accumulation point of the iterative sequence $\left\{\left(x^{k}, y^{k}, v^{k}\right)\right\}$ (see the proof of Theorem 1 in the sequel).

Define a penalty function $f_{r}(x, y)$ for the problem (6) by

$$
f_{r}(x, y)=f(x, y)-r \sum_{i \in I^{\mathcal{C}}}\left(v_{i}-F_{i}(x, y)\right)-r \sum_{i \in I^{\mathcal{C}}} \psi_{\varepsilon}\left(y_{i}, v_{i}\right),
$$

where $r>0$ is a penalty parameter. Similar to [29], we can convert the problem (6) to the following smoothing optimization only with inequality constraints:

$$
\begin{array}{cl}
\min & f_{r}(x, y) \\
\text { s.t. } & v_{i}-F_{i}(x, y) \leq 0, \quad i \in I^{\mathcal{C}}, \\
& \psi_{\varepsilon}\left(y_{i}, v_{i}\right) \leq 0, \quad i \in I^{\mathcal{C}}, \\
& g_{i}(x, y) \leq 0, \quad i \in I^{g} .
\end{array}
$$


For simplicity, we use the following notations throughout this paper:

$$
\begin{aligned}
& z=(x, y, v), \quad u=(x, y), \quad w=(y, v), \\
& d z=(d x, d y, d v), \quad d u=(d x, d y), \quad d w=(d y, d v), \\
& z^{k}=\left(x^{k}, y^{k}, v^{k}\right), \quad u^{k}=\left(x^{k}, y^{k}\right), \quad w^{k}=\left(y^{k}, v^{k}\right), \\
& d z^{k}=\left(d x^{k}, d y^{k}, d v^{k}\right), \quad d u^{k}=\left(d x^{k}, d y^{k}\right), \quad d w^{k}=\left(d y^{k}, d v^{k}\right), \\
& e_{I}=(1,1, \ldots, 1)^{T} \in \mathbb{R}^{2 m+m_{g}}, \quad e_{I^{\mathcal{C}}}=(1,1, \ldots, 1)^{T} \in \mathbb{R}^{m}, \\
& X:=\left\{z \in \mathbb{R}^{n+2 m} \mid v_{i}-F_{i}(x, y)=0, i \in I^{\mathcal{C}} ; \psi_{\varepsilon}\left(y_{i}, v_{i}\right)=0, i \in I^{\mathcal{C}} ; g_{i}(x, y) \leq 0, i \in I^{g}\right\}, \\
& \widetilde{X}:=\left\{z \in \mathbb{R}^{n+2 m} \mid v_{i}-F_{i}(x, y) \leq 0, i \in I^{\mathcal{C}} ; \psi_{\varepsilon}\left(y_{i}, v_{i}\right) \leq 0, i \in I^{\mathcal{C}} ; g_{i}(x, y) \leq 0, i \in I^{g}\right\}, \\
& \widetilde{X}_{0}:=\left\{z \in \mathbb{R}^{n+2 m} \mid v_{i}-F_{i}(x, y)<0, i \in I^{\mathcal{C}} ; \psi_{\varepsilon}\left(y_{i}, v_{i}\right)<0, i \in I^{\mathcal{C}} ; g_{i}(x, y)<0, i \in I^{g}\right\}, \\
& \varphi(x, y)=\max \left\{0 ; g_{i}(x, y), i \in I^{g}\right\}, \quad I(x, y)=\left\{i \in I^{g} \mid g_{i}(x, y)=\varphi(x, y)\right\}, \\
& I_{0}^{F}(z)=\left\{i \in I^{\mathcal{C}} \mid v_{i}-F_{i}(x, y)=0\right\}, \quad I_{0}^{\Psi_{\varepsilon}}(z)=\left\{i \in I^{\mathcal{C}} \mid \psi_{\varepsilon}\left(y_{i}, v_{i}\right)=0\right\}, \\
& I_{0}^{g}(z)=\left\{i \in I^{g} \mid g_{i}(x, y)=0\right\} . \quad
\end{aligned}
$$

The following proposition shows the equivalence between the problem (6) and the problem (7).

Proposition 2 If $\left(z, \lambda^{r}\right)$ is a KKT pair of the problem (7), and $v-F(x, y)=0, \Psi_{\varepsilon}(y, v)=0$, then $(z, \lambda)$ with multiplier $\lambda=\lambda^{r}-r \tilde{e}$ is a KKT pair of the problem (6), where $\lambda^{r}:=$ $\left(\lambda_{F, I^{\mathcal{C}}}^{r}, \lambda_{\Psi_{\varepsilon}, I^{\mathcal{C}}}^{r}, \lambda_{g, I^{g}}^{r}\right), \lambda:=\left(\lambda_{F, I^{\mathcal{C}}}, \lambda_{\Psi_{\varepsilon}, I^{\mathcal{C}}}, \lambda_{g, I^{g}}\right), \tilde{e}=\left(1, \ldots, 1_{m t h}, 1, \ldots, 1_{(2 m) t h}, 0, \ldots, 0_{\left(2 m+m_{g}\right) t h}\right)^{\mathrm{T}}$.

Proof Since $\left(z, \lambda^{r}\right)$ is a KKT pair of the problem (7), the vector pair $\left(z, \lambda^{r}\right)$ satisfies the following relations:

$$
\begin{aligned}
& \nabla_{z} f_{r}(x, y)+\nabla_{z}(v-F(x, y)) \lambda_{F, I^{\mathcal{C}}}^{r}+\nabla_{z} \Psi_{\varepsilon}(y, v) \lambda_{\Psi_{\varepsilon}, I^{\mathcal{C}}}^{r}+\nabla_{z} g(x, y) \lambda_{g, I^{g}}^{r}=0, \\
& \lambda_{F, i}^{r} \geq 0, v_{i}-F_{i}(x, y) \leq 0, \quad \lambda_{F, i}^{r}\left(v_{i}-F_{i}(x, y)\right)=0, \quad i \in I^{\mathcal{C}} \\
& \lambda_{\Psi_{\varepsilon}, i}^{r} \geq 0, \psi_{\varepsilon}\left(y_{i}, v_{i}\right) \leq 0, \quad \lambda_{\Psi_{\varepsilon}, i}^{r} \psi_{\varepsilon}\left(y_{i}, v_{i}\right)=0, \quad i \in I^{\mathcal{C}} \\
& \lambda_{g, i}^{r} \geq 0, g_{i}(x, y) \leq 0, \quad \lambda_{g, i}^{r} g_{i}(x, y)=0, \quad i \in I^{g} .
\end{aligned}
$$

Note that

$$
\nabla_{z} f_{r}(x, y)=\nabla_{z} f(x, y)-r \sum_{i \in I^{\mathcal{C}}} \nabla_{z}\left(v_{i}-F_{i}(x, y)\right)-r \sum_{i \in I^{\mathcal{C}}} \nabla_{z} \psi_{\varepsilon}\left(y_{i}, v_{i}\right)
$$

we get

$$
\nabla_{z} f(x, y)+\nabla_{z}(v-F(x, y))\left(\lambda_{F, I^{\mathcal{C}}}^{r}-r e_{I^{\mathcal{C}}}\right)+\nabla_{z} \Psi_{\varepsilon}(y, v)\left(\lambda_{\Psi_{\varepsilon}, I^{\mathcal{C}}}^{r}-r e_{I^{\mathcal{C}}}\right)+\nabla_{z} g(x, y) \lambda_{g, I^{g}}^{r}=0 .
$$

Let $\lambda_{F, I^{\mathcal{C}}}=\lambda_{F, I^{\mathcal{C}}}^{r}-r e_{I^{\mathcal{C}}}, \lambda_{\Psi_{\varepsilon}, I^{\mathcal{C}}}=\lambda_{\Psi_{\varepsilon}, I^{\mathcal{C}}}^{r}-r e_{I^{\mathcal{C}}}, \lambda_{g, I^{g}}=\lambda_{g, I^{g}}^{r}$, we obtain

$$
\nabla_{z} f(x, y)+\nabla_{z}(v-F(x, y)) \lambda_{F, I^{\mathcal{C}}}+\nabla_{z} \Psi_{\varepsilon}(y, v) \lambda_{\Psi_{\varepsilon}, I^{\mathcal{C}}}+\nabla_{z} g(x, y) \lambda_{g, I^{g}}=0
$$


In view of $v-F(x, y)=0, \Psi_{\varepsilon}(y, v)=0, g(x, y) \leq 0$, and

$$
\lambda_{g, i} \geq 0, \quad \lambda_{g, i} g_{i}(x, y)=0, \quad i \in I^{g} .
$$

Let $\lambda:=\left(\lambda_{F, I^{C}}, \lambda_{\Psi_{\varepsilon}, I^{C}}, \lambda_{g, I^{g}}\right)=\lambda^{r}-r \tilde{e}$, so $(z, \lambda)$ is a KKT pair of the problem (6).

\section{Description of the algorithm}

For the sake of theoretical analysis, we make a basic assumption throughout this paper.

\section{Assumption 1}

(1) For any $(x, y) \in \mathbb{R}^{n+m}$, the matrix $\nabla_{y} F(x, y)$ is a $P_{0}$ matrix, i.e., all the principal minors of $\nabla_{y} F(x, y)$ are nonnegative.

(2) For any $(x, y) \in \mathbb{R}^{n+m}$, the submatrix $\left(\nabla_{y} F(x, y)\right)_{J^{*} J^{*}}$ of the matrix $\nabla_{y} F(x, y)$ is nonsingular, where the index set $J^{*}=\left\{i \in I^{C} \mid \psi_{\varepsilon, y_{i}}^{\prime}\left(y_{i}, v_{i}\right)=0\right\}$.

In order to construct the coefficient matrix of linear equations conveniently, denote

$$
\Omega_{J}:=\Omega_{J}(z, \varepsilon)=\left(\begin{array}{c}
v-F(x, y) \\
\Psi_{\varepsilon}(y, v) \\
g_{J}(x, y)
\end{array}\right) \text {, }
$$

and denote by $A_{J}$ the gradient matrix of $\Omega_{J}$, that is,

$$
A_{J}:=A_{J}(z, \varepsilon)=\left(\begin{array}{ccc}
-\nabla_{x} F(x, y) & 0 & \nabla_{x} g_{J}(x, y) \\
-\nabla_{y} F(x, y) & \nabla_{y} \Psi_{\varepsilon}(y, v) & \nabla_{y} g_{J}(x, y) \\
E_{m} & \nabla_{\nu} \Psi_{\varepsilon}(y, v) & 0
\end{array}\right),
$$

where $J \subseteq I^{g}$, the diagonal matrix

$$
\nabla_{y} \Psi_{\varepsilon}(y, v)=\operatorname{diag}\left(\psi_{\varepsilon, y_{i}}^{\prime}\left(y_{i}, v_{i}\right), i \in I^{C}\right), \quad \nabla_{\nu} \Psi_{\varepsilon}(y, v)=\operatorname{diag}\left(\psi_{\varepsilon, v_{i}}^{\prime}\left(y_{i}, v_{i}\right), i \in I^{C}\right)
$$

Define the matrix

$$
U:=U(z, \varepsilon)=\left(\begin{array}{cc}
-\nabla_{y} F(x, y) & \nabla_{y} \Psi_{\varepsilon}(y, v) \\
E_{m} & \nabla_{\nu} \Psi_{\varepsilon}(y, v)
\end{array}\right) .
$$

Similar to the proof in $[30,31]$, we can prove that the following proposition is true.

\section{Proposition 3}

(1) Suppose that the matrix $U$ is nonsingular. Then the matrix $A_{I(x, y)}$ is full of column rank, if and only if the matrix

$$
\nabla_{x} g_{I(x, y)}(x, y)-\nabla_{x} F(x, y)\left(U^{-1}\right)_{m} \nabla_{y} g_{I(x, y)}(x, y)
$$

is full of column rank, where $\left(U^{-1}\right)_{m}$ is the mth-order principal submatrix of $U^{-1}$, which consists of the first $m$ rows and $m$ columns of $U^{-1}$.

(2) Suppose that Assumption 1 holds, then the matrix $U$ is nonsingular. 
Assumption 2 For any $z \in \mathbb{R}^{n+2 m}$, the matrix

$$
\nabla_{x} g_{I(x, y)}(x, y)-\nabla_{x} F(x, y)\left(U^{-1}\right)_{m} \nabla_{y} g_{I(x, y)}(x, y)
$$

is full of column rank.

Remark 1 According to Proposition 3, if Assumption 1 is true, then Assumption 2 is equivalent to the LICQ of the problem (7).

Based on Proposition 2, we know that if one can construct an efficient algorithm for the problem (7) and adjust penalty parameter $r$ to force the iterate to asymptotically satisfy $v-F(x, y)=0, \Psi_{\varepsilon}(y, v)=0$, then the solution to the problem (6) can be yielded.

Define the following optimal identification function:

$$
\rho(z, \lambda)=\|\Phi(z, \lambda)\|^{\vartheta}
$$

where

$$
\Phi(z, \lambda)=\left(\begin{array}{c}
\nabla_{z} L(z, \lambda) \\
v-F(x, y) \\
\Psi_{\varepsilon}(y, v) \\
\min \left\{-g(x, y), \lambda_{\left.g, I^{g}\right\}}\right.
\end{array}\right),
$$

and $\lambda=\left(\lambda_{F, I^{\mathcal{C}}}, \lambda_{\Psi_{\varepsilon}, \mathcal{I}^{\mathcal{C}}}, \lambda_{g, I^{g}}\right)$, the parameter $\vartheta \in(0,1),\|\cdot\|$ is the Euclidean norm, and we have the Lagrangian function

$$
L(z, \lambda)=f(x, y)+\sum_{i \in I^{\mathcal{C}}} \lambda_{F, i}\left(v_{i}-F_{i}(x, y)\right)+\sum_{i \in I^{\mathcal{C}}} \lambda_{\Psi_{\varepsilon}, i} \psi_{\varepsilon}\left(y_{i}, v_{i}\right)+\sum_{i \in I^{g}} \lambda_{g, i} g_{i}(x, y) .
$$

Obviously, $\rho$ is nonnegative and continuous. It follows from [32] that $\rho\left(z^{*}, \lambda^{*}\right)=0$ if and only if $\left(z^{*}, \lambda^{*}\right)$ is a KKT pair of the problem (6).

For the current iterate $z^{k} \in \widetilde{X}_{0}$, we define the corresponding multiplier vector $\lambda^{k}=$ $\left(\lambda_{F, I^{\mathcal{C}}}^{k}, \lambda_{\Psi_{\varepsilon}, I^{\mathcal{C}}}^{k}, \lambda_{g, I^{I}}^{k}\right)$ in (9) by

$$
\lambda^{0}=s^{0}, \quad \lambda^{k}=\widehat{\lambda}^{k-1}-r_{k-1} \tilde{e}, \quad k \geq 1,
$$

where $s^{0}>0$, and $\left(\widehat{\lambda}^{k-1}, r_{k-1}\right)$ is computed in the previous $(k-1)$ th iteration, we construct the working set $I_{k}$ as follows:

$$
I_{k}=\left\{i \in I^{g} \mid g_{i}\left(x^{k}, y^{k}\right)+\rho\left(z^{k}, \lambda^{k}\right) \geq 0\right\} .
$$

When $\left(z^{k}, \lambda^{k}\right)$ is sufficiently close to a KKT pair $\left(z^{*}, \lambda^{*}\right)$ of the problem (6), and the second-order sufficient conditions and the MFCQ hold at $\left(z^{*}, \lambda^{*}\right)$, the set $I_{k}$ equals the precise identification set $I_{0}^{g}\left(z^{*}\right)$. Therefore, only inequality constraints associated with the working set $I_{k}$ need to be considered. 
For the current iterate $z^{k} \in \mathbb{R}^{n+2 m}$, the first SLE in our algorithm is constructed as follows:

$$
\text { SLE1: } \quad M_{I_{k}}\left(\begin{array}{l}
d z \\
\lambda_{I_{k}}
\end{array}\right)=\left(\begin{array}{c}
-\nabla_{z} f_{r_{k}}\left(x^{k}, y^{k}\right) \\
0
\end{array}\right) \text {, }
$$

where the coefficient matrix $M_{I_{k}}$ is defined by

$$
M_{I_{k}}:=M_{I_{k}}\left(z^{k}, S_{I_{k}}\right)=\left(\begin{array}{cc}
H_{k} & A_{I_{k}} \\
S_{I_{k}}\left(A_{I_{k}}\right)^{\mathrm{T}} & G_{I_{k}}
\end{array}\right)
$$

with

$$
G_{I_{k}}=\operatorname{diag}\left(v^{k}-F\left(x^{k}, y^{k}\right), \Psi_{\varepsilon}\left(y^{k}, v^{k}\right), g_{I_{k}}\left(x^{k}, y^{k}\right)\right)
$$

the matrix $H_{k} \in \mathbb{R}^{n+2 m}$ is an approximation of the Lagrangian Hessian, the matrix $S_{I_{k}}:=$ $\operatorname{diag}\left(s_{I_{k}}^{k}\right)$, where the vector $s_{I_{k}}^{k}:=\left(s_{F, I^{\mathcal{C}}}^{k}, s_{\Psi_{\varepsilon}, I^{\mathcal{C}}}^{k}, s_{g, I_{k}}^{k}\right) \in \mathbb{R}^{2 m+\left|I_{k}\right|}$ is an approximation of $\lambda_{I_{k}}^{k}:=$ $\left(\lambda_{F, I^{\mathcal{C}}}^{k}, \lambda_{\Psi_{\varepsilon, I}}^{k}, \lambda_{g, I_{k}}^{k}\right)$.

It is not difficult to prove that the following result is true.

Lemma 1 Suppose that Assumptions 1-2 hold, and the symmetric matrix $H_{k}$ satisfies the following relation:

$$
\begin{aligned}
H_{k} \succ & \sum_{i \in I^{\mathcal{C}}} \frac{s_{F, i}^{k}}{v_{i}^{k}-F_{i}\left(x^{k}, y^{k}\right)} \nabla_{z}\left(v_{i}^{k}-F_{i}\left(x^{k}, y^{k}\right)\right) \nabla_{z}\left(v_{i}^{k}-F_{i}\left(x^{k}, y^{k}\right)\right)^{\mathrm{T}} \\
& +\sum_{i \in I^{\mathcal{C}}} \frac{s_{\Psi_{\varepsilon}, i}^{k}}{\psi_{\varepsilon}\left(y_{i}^{k}, v_{i}^{k}\right)} \nabla_{z} \psi_{\varepsilon}\left(y_{i}^{k}, v_{i}^{k}\right) \nabla_{z} \psi_{\varepsilon}\left(y_{i}^{k}, v_{i}^{k}\right)^{\mathrm{T}} \\
& +\sum_{i \in I_{k}} \frac{s_{g, i}^{k}}{g_{i}\left(x^{k}, y^{k}\right)} \nabla_{z} g_{i}\left(x^{k}, y^{k}\right) \nabla_{z} g_{i}\left(x^{k}, y^{k}\right)^{\mathrm{T}}
\end{aligned}
$$

then the coefficient matrix $M_{I_{k}}$ defined by (13) is invertible, where the matrix order $A \succ B$ means that $A-B$ is positive definite.

We now describe our algorithm as follows.

\section{Algorithm A Step 0. (Initialization)}

Choose an initial point $z^{0} \in \widetilde{X}_{0} ; \alpha \in\left(0, \frac{1}{2}\right) ; \vartheta, \beta, \theta \in(0,1) ; r_{0}, \varepsilon_{0}, C, v, \gamma_{1}, \gamma_{2}, \gamma_{3}, \gamma_{4}>0$; $\tau>1 ; q>2$; termination accuracy $\epsilon>0$; the vector $s^{0}:=\left(s_{F, I^{\mathcal{C}}}^{0}, s_{\Psi_{\varepsilon}, I^{\mathcal{C}}}^{0}, s_{g, I^{g}}^{0}\right) \in \mathbb{R}^{2 m+m_{g}}$ with $s_{i}^{0} \in\left[s_{\min }, s_{\max }\right]$, where $s_{\max }>s_{\min }>0$. Set $k:=0$.

Step 1. (Compute the working set)

Yield $\lambda^{k}$ by (10), then compute $\rho\left(z^{k}, \lambda^{k}\right)$ by (9). If $\rho\left(z^{k}, \lambda^{k}\right) \leq \epsilon$, and $I^{\mathcal{C}}\left(y^{k}, v^{k}, \varepsilon_{k}\right)=\emptyset$, then $z^{k}$ is a KKT point of the MPCC (1), stop; otherwise, compute the working set $I_{k}$ by (11).

Step 2. (Yield a matrix $H_{k}$ )

Generate a symmetric matrix $H_{k}$ such that it is an approximation of the Lagrangian Hessian of the problem (7) and satisfies the condition (14).

Step 3. (Generate a search direction) 
(1) Solve the first SLE1 (12). Let its solution be $\left(\widehat{d z}^{k}, \widehat{\lambda}_{I_{k}}^{k}\right)$, with $\widehat{\lambda}_{I_{k}}^{k}:=\left(\widehat{\lambda}_{F, I^{\mathcal{C}}}^{k}, \widehat{\lambda}_{\Psi_{\varepsilon}, I^{\mathcal{C}}}^{k}, \widehat{\lambda}_{g, I_{k}}^{k}\right) \in \mathbb{R}^{2 m+\left|I_{k}\right|}$. Set $\widehat{\lambda}^{k}:=\left(\widehat{\lambda}_{F, I^{\mathcal{C}}}^{k}, \widehat{\lambda}_{\Psi_{\varepsilon}, I^{\mathcal{C}}}^{k}, \widehat{\lambda}_{g, I^{g}}^{k}\right)$, where $\widehat{\lambda}_{g, I^{g}}^{k}:=\left(\widehat{\lambda}_{g, I_{k}}^{k}, 0_{I^{g} \backslash I_{k}}\right)$.

(2) If the following conditions hold:

(i) $\left\|\widehat{d z}^{k}\right\| \leq \gamma_{1}$

(ii) $\widehat{\lambda}^{k} \geq-\gamma_{2} e_{I}$,

(iii) $\widehat{\lambda}_{F, I}^{k} \mathcal{\ngtr} \gamma_{3} e_{I} \mathcal{c}$,

(iv) $\widehat{\lambda}_{\Psi_{s}, I^{c}}^{k} \ngtr \gamma_{4} e_{I} \mathcal{C}$,

(v) $I^{\mathcal{C}}\left(y^{k}, v^{k}, \varepsilon_{k}\right) \neq \emptyset$, then update the parameter $r_{k}$ by $r_{k+1}=\tau r_{k}$, set $z^{k+1}=z^{k}$, $s^{k+1}=s^{k}, H_{k+1}=H_{k}, I_{k+1}=I_{k}, \varepsilon_{k+1}=\frac{1}{2} \varepsilon_{k}$, and go back to Step 3(1); otherwise, set $r_{k+1}=r_{k}$, go to Step 3(3).

(3) Find $\phi_{F, I^{\mathcal{C}}}^{k}=\left(\phi_{F, i}^{k}\right) \in \mathbb{R}^{m}, D_{\Psi_{\varepsilon}, I^{\mathcal{C}}}^{k}=\left(D_{\Psi_{\varepsilon}, i}^{k}\right) \in \mathbb{R}^{m}, Q_{g, I_{k}}^{k}=\left(Q_{g, i}^{k}\right) \in \mathbb{R}^{\left|I_{k}\right|}$, respectively, as follows:

$$
\begin{aligned}
\phi_{F, i}^{k} & :=\phi_{i}\left(z^{k}, \hat{\lambda}_{F, i}^{k}\right) \\
& =\min \left\{0,-\left(\max \left\{-\widehat{\lambda}_{F, i}^{k}, 0\right\}\right)^{v}-C\left(v_{i}^{k}-F_{i}\left(x^{k}, y^{k}\right)\right)\right\}, \quad i \in I^{\mathcal{C}}, \\
D_{\Psi_{\varepsilon}, i}^{k} & :=D_{i}\left(z^{k}, \widehat{\lambda}_{\Psi_{\varepsilon}, i}^{k}\right)=\min \left\{0,-\left(\max \left\{-\widehat{\lambda}_{\Psi_{\varepsilon}, i}^{k}, 0\right\}\right)^{v}-C \psi_{\varepsilon}\left(y_{i}^{k}, v_{i}^{k}\right)\right\}, \quad i \in I^{\mathcal{C}}, \\
Q_{g, i}^{k} & :=Q_{i}\left(z^{k}, \hat{\lambda}_{g, i}^{k}\right)=\min \left\{0,-\left(\max \left\{-\widehat{\lambda}_{g, i}^{k}, 0\right\}\right)^{v}-C g_{i}\left(x^{k}, y^{k}\right)\right\}, \quad i \in I_{k} .
\end{aligned}
$$

Denote $\varpi^{k}=\left(\phi_{F, I^{C}}^{k}, D_{\Psi_{\varepsilon}, I^{C}}^{k}, Q_{g, I_{k}}^{k}\right)$, then compute

$$
\begin{aligned}
\zeta^{k}= & \nabla_{z} f_{r_{k}}\left(x^{k}, y^{k}\right)^{\mathrm{T}} \widehat{d z}^{k}-\sum_{i \in I^{\mathcal{C}}} \frac{\widehat{\lambda}_{F, i}^{k}}{s_{F, i}^{k}} \phi_{F, i}^{k}-\sum_{i \in I^{\mathcal{C}}} \frac{\widehat{\lambda}_{\Psi_{\varepsilon}, i}^{k}}{s_{\Psi_{\varepsilon}, i}^{k}} D_{\Psi_{\varepsilon}, i}^{k}-\sum_{i \in I_{k}} \frac{\widehat{\lambda}_{g, i}^{k}}{s_{g, i}^{k}} Q_{g, i}^{k}, \\
b_{k}= & \left(\left\|\widehat{d z}^{k}\right\|^{q}+\left\|\varpi^{k}\right\|\right)\left(\sum_{i \in I^{\mathcal{C}}} \widehat{\lambda}_{F, i}^{k}+\sum_{i \in I^{\mathcal{C}}} \widehat{\lambda}_{\Psi_{\varepsilon} i}^{k}+\sum_{i \in I_{k}} \widehat{\lambda}_{g, i}^{k}\right) \\
& +\sum_{i \in I^{\mathcal{C}}} \frac{\widehat{\lambda}_{F, i}^{k}}{s_{F, i}^{k}} \phi_{F, i}^{k}+\sum_{i \in I^{\mathcal{C}}} \frac{\widehat{\lambda}_{\Psi_{\varepsilon}, i}^{k}}{s_{\Psi_{\varepsilon}, i}^{k}} D_{\Psi_{\varepsilon}, i}^{k}+\sum_{i \in I_{k}} \frac{\hat{\lambda}_{g, i}^{k}}{s_{g, i}^{k}} Q_{g, i}^{k}, \\
\varrho_{k}= & \begin{cases}1, & \text { if } b_{k} \leq 0, \\
\min \left\{\frac{(1-\theta)\left|\zeta^{k}\right|}{b_{k}}, 1\right\}, & \text { if } b_{k}>0 .\end{cases}
\end{aligned}
$$

(4) Solve the second SLE as follows:

$$
\text { SLE2: } \quad M_{I_{k}}\left(\begin{array}{c}
d z \\
\lambda_{I_{k}}
\end{array}\right)=\left(\begin{array}{c}
-\nabla_{z} f_{r_{k}}\left(x^{k}, y^{k}\right) \\
\eta_{I_{k}}^{k}
\end{array}\right) \text {, }
$$

where the perturbation vector $\eta_{I_{k}}^{k}=\left(\eta_{F, I}^{k}, \eta_{\Psi_{\varepsilon}, \mathcal{C}}^{k}, \eta_{g, I_{k}}^{k}\right)$ is determined by the following convex combination:

$$
\eta_{I_{k}}^{k}=\left(1-\varrho_{k}\right) \varpi^{k}+\varrho_{k}\left(-\left\|\widehat{d z}^{k}\right\|-\left\|\varpi^{k}\right\|\right) s_{I_{k}}^{k}
$$

with $s_{I_{k}}^{k}:=\left(s_{F, I^{\mathcal{C}}}^{k}, s_{\Psi_{\varepsilon}, I^{\mathcal{C}}}^{k}, s_{g, I_{k}}^{k}\right) \in \mathbb{R}^{2 m+\left|I_{k}\right|}$.

Let its solution be $\left(d z^{k}, \lambda_{I_{k}}^{k}\right)$ with $\lambda_{I_{k}}^{k}:=\left(\lambda_{F, I}^{k}, \lambda_{\Psi_{\varepsilon},}^{k} \mathcal{C}, \lambda_{g, I_{k}}^{k}\right) \in \mathbb{R}^{2 m+\left|I_{k}\right|}$. Set $\lambda^{k}:=\left(\lambda_{F, I^{\mathcal{C}}}^{k}, \lambda_{\Psi_{\varepsilon}, I^{\mathcal{C}}}^{k}, \lambda_{g, I^{g}}^{k}\right)$ with $\lambda_{g, I^{g}}^{k}=\left(\lambda_{g, I_{k}}^{k}, 0_{I^{g} \backslash I_{k}}\right)$. 
Step 4. (Perform line search) Compute the step size $t_{k}$, which is the first number $t$ of the sequence $\left\{1, \beta, \beta^{2}, \ldots\right\}$ satisfying

$$
\begin{aligned}
& f_{r_{k}}\left(z^{k}+t d z^{k}\right) \leq f_{r_{k}}\left(z^{k}\right)+\alpha t \nabla_{z} f_{r_{k}}\left(x^{k}, y^{k}\right)^{\mathrm{T}} d z^{k}, \\
& \left(v_{i}^{k}+t d v^{k}\right)-F_{i}\left(u^{k}+t d u^{k}\right)<0, \quad \forall i \in I^{\mathcal{C}}, \\
& \psi_{\varepsilon}\left(w_{i}^{k}+t d w^{k}\right)<0, \quad \forall i \in I^{\mathcal{C}}, \\
& g_{i}\left(u^{k}+t d u^{k}\right)<0, \quad \forall i \in I^{g} .
\end{aligned}
$$

Step 5. (Update) Let $z^{k+1}=z^{k}+t_{k} d z^{k}$, and compute

$$
\begin{aligned}
s_{i}^{k+1} & =\min \left\{\max \left\{\left\|d z^{k}\right\|^{2}+s_{\min }, \lambda_{i}^{k}\right\}, s_{\max }\right\}, \\
\xi_{k+1} & =\min \left\{\sqrt{\left(y_{i}^{k+1}\right)^{2}+\left(v_{i}^{k+1}\right)^{2}}, i \in I^{\mathcal{C}}\right\}, \\
\varepsilon_{k+1} & = \begin{cases}\varepsilon_{k}, & \text { if } \varepsilon_{k} \leq \xi_{k+1}, \\
\frac{1}{2} \varepsilon_{k}, & \text { otherwise. }\end{cases}
\end{aligned}
$$

Step 6. Let $k:=k+1$, and go back to Step 1 .

Remark 2 The correction technique of penalty parameter $r_{k}$ in Step 3(2) is from [33], when the conditions (i)-(ii) in Step 3(2) are satisfied, the current iteration point $z^{k}$ is close to a KKT point of the problem (7). However, the conditions (iii)-(iv) in Step 3(2) indicate that $z^{k}$ is far away from the feasible area of the problem (6), and the penalty parameter needs to be increased.

For convenience, denote

$$
\begin{aligned}
\Lambda_{k}= & H_{k}-\sum_{i \in I^{\mathcal{C}}} \frac{s_{F, i}^{k}}{v_{i}^{k}-F_{i}\left(x^{k}, y^{k}\right)} \nabla_{z}\left(v_{i}^{k}-F_{i}\left(x^{k}, y^{k}\right)\right) \nabla_{z}\left(v_{i}^{k}-F_{i}\left(x^{k}, y^{k}\right)\right)^{\mathrm{T}} \\
& -\sum_{i \in I^{\mathcal{C}}} \frac{s_{\Psi_{\varepsilon}, i}^{k}}{\psi_{\varepsilon}\left(y_{i}^{k}, v_{i}^{k}\right)} \nabla_{z} \psi_{\varepsilon}\left(y_{i}^{k}, v_{i}^{k}\right) \nabla_{z} \psi_{\varepsilon}\left(y_{i}^{k}, v_{i}^{k}\right)^{\mathrm{T}} \\
& -\sum_{i \in I_{k}} \frac{s_{g, i}^{k}}{g_{i}\left(x^{k}, y^{k}\right)} \nabla_{z} g_{i}\left(x^{k}, y^{k}\right) \nabla_{z} g_{i}\left(x^{k}, y^{k}\right)^{\mathrm{T}}
\end{aligned}
$$

Lemma 2 For the directions $\widehat{d z}^{k}$ and $d z^{k}$ found in Step 3(1)(4), the following conclusions hold:

$$
\begin{aligned}
& \nabla_{z} f_{r_{k}}\left(x^{k}, y^{k}\right)^{\mathrm{T}} \widehat{d z}^{k}=-\left(\widehat{d}^{k}\right)^{\mathrm{T}} \Lambda_{k} \widehat{d z}^{k} \leq 0, \quad \forall k \geq 0, \\
& \nabla_{z} f_{r_{k}}\left(x^{k}, y^{k}\right)^{\mathrm{T}} d z^{k} \leq \theta \zeta^{k} \leq 0, \quad \forall k \geq 0 .
\end{aligned}
$$

Furthermore, when iteration goes into Step 3(3)(4), we have $\widehat{d z}^{k} \neq 0$ and $\zeta^{k}<0$, so dz $z^{k}$ is a feasible descent direction of the problem (7) at $z^{k}$, hence, Algorithm A is well defined. 
Proof It follows from the SLE1 (12) that

$$
\begin{aligned}
& H_{k} \widehat{d z}^{k}+A_{I_{k}} \widehat{\lambda}_{I_{k}}^{k}=-\nabla_{z} f_{r_{k}}\left(x^{k}, y^{k}\right), \\
& S_{I_{k}}\left(A_{I_{k}}\right)^{\mathrm{T}} \widehat{d z}^{k}+G_{I_{k}} \widehat{\lambda}_{I_{k}}=0 .
\end{aligned}
$$

By (30)-(31), we have

$$
\begin{aligned}
\nabla_{z} f_{r_{k}}\left(x^{k}, y^{k}\right)= & \sum_{i \in I^{\mathcal{C}}} \frac{s_{F, i}^{k}}{v_{i}^{k}-F_{i}\left(x^{k}, y^{k}\right)} \nabla_{z}\left(v_{i}^{k}-F_{i}\left(x^{k}, y^{k}\right)\right) \nabla_{z}\left(v_{i}^{k}-F_{i}\left(x^{k}, y^{k}\right)\right)^{\mathrm{T}} \widehat{d z}^{k} \\
& +\sum_{i \in I^{\mathcal{C}}} \frac{s_{\Psi_{\varepsilon}, i}^{k}}{\psi_{\varepsilon}\left(y_{i}^{k}, v_{i}^{k}\right)} \nabla_{z} \psi_{\varepsilon}\left(y_{i}^{k}, v_{i}^{k}\right) \nabla_{z} \psi_{\varepsilon}\left(y_{i}^{k}, v_{i}^{k}\right)^{\mathrm{T}} \widehat{d z}^{k} \\
& +\sum_{i \in I_{k}} \frac{s_{g, i}^{k}}{g_{i}\left(x^{k}, y^{k}\right)} \nabla_{z} g_{i}\left(x^{k}, y^{k}\right) \nabla_{z} g_{i}\left(x^{k}, y^{k}\right)^{\mathrm{T}} \widehat{d z}^{k}-H_{k} \widehat{d z}^{k}
\end{aligned}
$$

which indicates $\nabla_{z} f_{r_{k}}\left(x^{k}, y^{k}\right)^{\mathrm{T}} \widehat{d z}^{k}=-\left(\widehat{d z}^{k}\right)^{\mathrm{T}} \Lambda_{k} \widehat{d z}^{k} \leq 0$.

By (15)-(18), we obtain

$$
\phi_{F, i} \widehat{\lambda}_{F, i}^{k} \geq 0, \quad i \in I^{\mathcal{C}} ; \quad D_{\Psi_{\varepsilon}, i} \widehat{\lambda}_{\Psi_{\varepsilon}, i}^{k} \geq 0, \quad i \in I^{\mathcal{C}} ; \quad Q_{g, i} \widehat{\lambda}_{g, i}^{k} \geq 0, \quad i \in I_{k}
$$

It follows from (18) and (32) that

$$
\zeta_{k} \leq \nabla_{z} f_{r_{k}}\left(x^{k}, y^{k}\right)^{\mathrm{T}} \widehat{d z}^{k} \leq 0
$$

Taking into account SLE1 (12) and SLE2 (21), we have

$$
\begin{aligned}
& H_{k} \widehat{d z}^{k}+A_{I_{k}} \widehat{\lambda}_{I_{k}}^{k}=-\nabla_{z} f_{r_{k}}\left(x^{k}, y^{k}\right), \\
& S_{I_{k}}\left(A_{I_{k}}\right)^{\mathrm{T}} \widehat{d d}^{k}+G_{I_{k}} \widehat{\lambda}_{I_{k}}^{k}=0, \\
& H_{k} d z^{k}+A_{I_{k}} \lambda_{I_{k}}^{k}=-\nabla_{z} f_{r_{k}}\left(x^{k}, y^{k}\right), \\
& S_{I_{k}}\left(A_{I_{k}}\right)^{\mathrm{T}} d z^{k}+G_{I_{k}} \lambda_{I_{k}}^{k}=\eta_{I_{k}}^{k} .
\end{aligned}
$$

It follows from (34) and (36) as well as the symmetry of the matrix $H_{k}$ that

$$
\begin{aligned}
& \nabla_{z} f_{r_{k}}\left(x^{k}, y^{k}\right)^{\mathrm{T}} d z^{k}-\nabla_{z} f_{r_{k}}\left(x^{k}, y^{k}\right)^{\mathrm{T}} \widehat{d}^{k} \\
& =\left(\widehat{d}^{k}\right)^{\mathrm{T}} A_{I_{k}} \lambda_{I_{k}}^{k}-\left(d z^{k}\right)^{\mathrm{T}} A_{I_{k}} \widehat{\lambda}_{I_{k}}^{k} \\
& \quad=\left(\lambda_{I_{k}}^{k}\right)^{\mathrm{T}}\left(A_{I_{k}}\right)^{\mathrm{T}} \widehat{d z}^{k}-\left(\hat{\lambda}_{I_{k}}^{k}\right)^{\mathrm{T}}\left(A_{I_{k}}\right)^{\mathrm{T}} d z^{k} .
\end{aligned}
$$

From (35) and (37), we have

$$
\begin{aligned}
& \left(\lambda_{I_{k}}^{k}\right)^{\mathrm{T}}\left(A_{I_{k}}\right)^{\mathrm{T}} \widehat{d d z}^{k}-\left(\widehat{\lambda}_{I_{k}}^{k}\right)^{\mathrm{T}}\left(A_{I_{k}}\right)^{\mathrm{T}} d z^{k} \\
& =-\sum_{i \in I^{\mathcal{C}}} \frac{\widehat{\lambda}_{F, i}^{k} \eta_{F, i}^{k}}{s_{F, i}^{k}}-\sum_{i \in I^{\mathcal{C}}} \frac{\widehat{\lambda}_{\Psi_{\varepsilon}, i}^{k} \eta_{\Psi_{\varepsilon}, i}^{k}}{s_{\Psi_{\varepsilon}, i}^{k}}-\sum_{i \in I_{k}} \frac{\widehat{\lambda}_{g, i}^{k} \eta_{g, i}^{k}}{s_{g, i}^{k}} .
\end{aligned}
$$


By (18) and (19), one gets

$$
\begin{aligned}
& \nabla_{z} f_{r_{k}}\left(x^{k}, y^{k}\right)^{\mathrm{T}} d z^{k} \\
& =\nabla_{z} f_{r_{k}}\left(x^{k}, y^{k}\right)^{\mathrm{T}} \widehat{d z}^{k}-\sum_{i \in I^{\mathcal{C}}} \frac{\widehat{\lambda}_{F, i}^{k} \eta_{F, i}^{k}}{s_{F, i}^{k}}-\sum_{i \in I^{\mathcal{C}}} \frac{\widehat{\lambda}_{\Psi_{\varepsilon}, i}^{k} \eta_{\Psi_{\varepsilon}, i}^{k}}{s_{\Psi_{\varepsilon}, i}^{k}}-\sum_{i \in I_{k}} \frac{\widehat{\lambda}_{g, i}^{k} \eta_{g, i}^{k}}{s_{g, i}^{k}} \\
& =\nabla_{z} f_{r_{k}}\left(x^{k}, y^{k}\right)^{\mathrm{T}} \widehat{d z}^{k}-\sum_{i \in I^{\mathcal{C}}} \frac{\widehat{\lambda}_{F, i}^{k}}{s_{F, i}^{k}}\left[\left(1-\varrho_{k}\right) \phi_{F, i}^{k}+\varrho_{k}\left(-\left\|\widehat{d z}^{k}\right\|^{q}-\left\|\varpi^{k}\right\|\right) s_{F, i}^{k}\right] \\
& -\sum_{i \in I^{\mathcal{C}}} \frac{\widehat{\lambda}_{\Psi_{\varepsilon}, i}^{k}}{s_{\Psi_{\varepsilon}, i}^{k}}\left[\left(1-\varrho_{k}\right) D_{\Psi_{\varepsilon}, i}^{k}+\varrho_{k}\left(-\left\|\widehat{d z}^{k}\right\|^{q}-\left\|\varpi^{k}\right\|\right) s_{\Psi_{\varepsilon}, i}^{k}\right] \\
& -\sum_{i \in I_{k}} \frac{\widehat{\lambda}_{g, i}^{k}}{s_{g, i}^{k}}\left[\left(1-\varrho_{k}\right) Q_{g, i}^{k}+\varrho_{k}\left(-\left\|\widehat{d z}^{k}\right\|^{q}-\left\|\varpi^{k}\right\|\right) s_{g, i}^{k}\right] \\
& =\left(\nabla_{z} f_{r_{k}}\left(x^{k}, y^{k}\right)^{\mathrm{T}} \widehat{d z}^{k}-\sum_{i \in I^{\mathcal{C}}} \frac{\widehat{\lambda}_{F, i}^{k}}{s_{F, i}^{k}} \phi_{F, i}^{k}-\sum_{i \in I^{\mathcal{C}}} \frac{\widehat{\lambda}_{\Psi_{\varepsilon}, i}^{k}}{s_{\Psi_{\varepsilon}, i}^{k}} D_{\Psi_{\varepsilon}, i}^{k}-\sum_{i \in I_{k}} \frac{\widehat{\lambda}_{g, i}^{k}}{s_{g, i}^{k}} Q_{g, i}^{k}\right) \\
& +\varrho_{k}\left[\sum_{i \in I^{\mathcal{C}}} \frac{\widehat{\lambda}_{F, i}^{k}}{s_{F, i}^{k}} \phi_{F, i}^{k}+\sum_{i \in I^{\mathcal{C}}} \widehat{\lambda}_{F, i}^{k}\left(\left\|\widehat{d z}^{k}\right\|^{q}+\left\|\varpi^{k}\right\|\right)\right] \\
& +\varrho_{k}\left[\sum_{i \in I^{\mathcal{C}}} \frac{\widehat{\lambda}_{\Psi_{\varepsilon}, i}^{k}}{s_{\Psi_{\varepsilon}, i}^{k}} D_{\Psi_{\varepsilon}, i}^{k}+\sum_{i \in I^{\mathcal{C}}} \widehat{\lambda}_{\Psi_{\varepsilon}, i}^{k}\left(\left\|\widehat{d z}^{k}\right\|^{q}+\left\|\varpi^{k}\right\|\right)\right] \\
& +\varrho_{k}\left[\sum_{i \in I_{k}} \frac{\widehat{\lambda}_{g, i}^{k}}{s_{g, i}^{k}} Q_{g, i}^{k}+\sum_{i \in I_{k}} \widehat{\lambda}_{g, i}^{k}\left(\left\|\widehat{d z}^{k}\right\|^{q}+\left\|\varpi^{k}\right\|\right)\right] \\
& =\zeta^{k}+\varrho_{k} b_{k} \text {. }
\end{aligned}
$$

In view of (20), if $b_{k} \leq 0$, then $\varrho_{k} b_{k}=b_{k} \leq 0$. Furthermore, we have $\zeta^{k}+\varrho_{k} b_{k} \leq \zeta^{k} \leq$ $\theta \zeta^{k}$. If $b_{k}>0$, then we have $\zeta^{k}+\varrho_{k} b_{k}=\zeta^{k}+\min \left\{(1-\theta)\left|\zeta^{k}\right|, b_{k}\right\} \leq \zeta^{k}+(\theta-1) \zeta^{k}=\theta \zeta^{k}$. In conclusion, we obtain $\zeta^{k}+\varrho_{k} b_{k} \leq \theta \zeta^{k}$. From (33) and (38), it follows that

$$
\nabla_{z} f_{r_{k}}\left(x^{k}, y^{k}\right)^{\mathrm{T}} d z^{k}=\zeta^{k}+\varrho_{k} b_{k} \leq \theta \zeta^{k} \leq 0
$$

which indicates that the inequality (29) is true.

If $\widehat{d z}^{k}=0$, taking into account SLE1 (12), from $v^{k}-F\left(x^{k}, y^{k}\right)<0, \Psi_{\varepsilon}\left(y^{k}, v^{k}\right)<0, g\left(x^{k}, y^{k}\right)<$ 0 , we have $\widehat{\lambda}_{I_{k}}^{k}=0$, so the iterate $k$ does not go into Step 3(3)(4). Thus, $\widehat{d z}^{k} \neq 0$ when the iterative process goes into Step 3(3)(4).

By $\nabla_{z} f_{r_{k}}\left(x^{k}, y^{k}\right)^{\mathrm{T}} d z^{k} \leq 0$, we know that $d z^{k}$ is a feasible descent direction of the problem (7) at point $z^{k}$. Together with $v^{k}-F\left(x^{k}, y^{k}\right)<0, \Psi_{\varepsilon}\left(y^{k}, v^{k}\right)<0, g\left(x^{k}, y^{k}\right)<0$ and $\zeta^{k}<0$, Step 4 in Algorithm A can be finished by finite calculations, hence Algorithm A is well defined. $\square$

\section{Global convergence}

In this paper, we assume that Algorithm A generates an infinite iteration sequence $\left\{z^{k}\right\}$. For the sake of convenience, let $z^{*}$ be an accumulation point of the iteration sequence $\left\{z^{k}\right\}$. First, we show that the penalty parameter $r_{k}$ can be augmented only in a finite number of steps. Then we prove that $z^{*}$ is a KKT point of the problem (7). Finally, we show that $z^{*}$ 
is a KKT point of the MPCC (1). More precisely, we show that Algorithm A is globally convergent. For this purpose, the following assumptions are necessary.

Assumption 3 Suppose that the sequences $\left\{H_{k}\right\}$ and $\left\{z^{k}\right\}$ are all bounded, each accumulation point of $\left\{z^{k}\right\}$ satisfies LLSC (2). Assume that there exists a positive constant $\sigma$ such that

$$
d^{\mathrm{T}} \Lambda_{k} d \geq \sigma\|d\|^{2}, \quad \forall k, \forall d \in \mathbb{R}^{n+2 m} .
$$

Assumption 4 For any $z \in \widetilde{X}$, if $z \notin X$, then no scalars $\lambda_{F, i} \geq 0, i \in I_{0}^{F}(z) ; \lambda_{\Psi_{\varepsilon}, i} \geq 0, i \in I_{0}^{\Psi_{\varepsilon}}(z)$ and $\lambda_{g, i} \geq 0, i \in I_{0}^{g}(z)$, exist such that

$$
\begin{aligned}
& \sum_{i \in I^{\mathcal{C}}} \nabla_{z}\left(v_{i}-F_{i}(x, y)\right)+\sum_{i \in I^{\mathcal{C}}} \nabla_{z} \psi_{\varepsilon}\left(y_{i}, v_{i}\right) \\
& \quad=\sum_{i \in I_{0}^{F}(z)} \lambda_{F, i} \nabla_{z}\left(v_{i}-F_{i}(x, y)\right)+\sum_{i \in I_{0}^{\Psi_{\varepsilon}}(z)} \lambda_{\Psi_{\varepsilon}, i} \nabla_{z} \psi_{\varepsilon}\left(y_{i}, v_{i}\right)+\sum_{i \in I_{0}^{g}(z)} \lambda_{g, i} \nabla_{z} g_{i}(x, y) .
\end{aligned}
$$

Lemma 3 Suppose that Assumptions 1-4 hold, then the penalty parameter $r_{k}$ can be augmented only in a finite number of steps.

Proof By contradiction, suppose that $r_{k}$ is increased infinitely many times, i.e., there exists an infinite index set $K$ such that $r_{k+1}>r_{k}$ for any $k \in K$. The increase of $r_{k}$ must satisfy the following conditions for any $k \in K$ :
(i) $\left\|\widehat{d z}^{k}\right\| \leq \gamma_{1}$;
(ii) $\widehat{\lambda}^{k} \geq-\gamma_{2} e_{I}$;
(iii) $\widehat{\lambda}_{F, I}^{k} \ngtr \ngtr \gamma_{3} e_{I} \mathcal{C}$;
(iv) $\widehat{\lambda}_{\Psi_{\varepsilon}, I^{\mathcal{C}}}^{k} \ngtr \gamma_{4} e_{I} \mathcal{C}$;
(v) $I^{C}\left(y^{k}, v^{k}, \varepsilon_{k}\right) \neq \emptyset$.

Denote

$$
\begin{aligned}
A_{I^{g}}^{k} & =\left(\begin{array}{ccc}
-\nabla_{x} F\left(x^{k}, y^{k}\right) & 0 & \nabla_{x} g\left(x^{k}, y^{k}\right) \\
-\nabla_{y} F\left(x^{k}, y^{k}\right) & \nabla_{y} \Psi_{\varepsilon}\left(y^{k}, v^{k}\right) & \nabla_{y} g\left(x^{k}, y^{k}\right) \\
E_{m} & \nabla_{\nu} \Psi_{\varepsilon}\left(y^{k}, v^{k}\right) & 0
\end{array}\right), \\
G_{I^{g}}^{k} & =\operatorname{diag}\left(v^{k}-F\left(x^{k}, y^{k}\right), \Psi_{\varepsilon}\left(y^{k}, v^{k}\right), g\left(x^{k}, y^{k}\right)\right),
\end{aligned}
$$

and $S_{I^{g}}^{k}=\operatorname{diag}\left(s_{I^{g}}^{k}\right)$, where $s_{I g}^{k}=\left(s_{F, \mathcal{C}^{\mathcal{C}}}^{k}, s_{\Psi_{\varepsilon}, \mathcal{C}^{\mathcal{C}}}^{k}, s_{g, I^{g}}^{k}\right)$. According to the SLE

$$
\left(\begin{array}{cc}
H_{k} & A_{I^{g}}^{k} \\
S_{I^{g}}^{k}\left(A_{I^{g}}^{k}\right)^{\mathrm{T}} & G_{I^{g}}^{k}
\end{array}\right)\left(\begin{array}{c}
\widehat{d z}^{k} \\
\widehat{\lambda}^{k}
\end{array}\right)=\left(\begin{array}{c}
-\nabla_{z} f_{r_{k}}\left(x^{k}, y^{k}\right) \\
0
\end{array}\right),
$$

we have

$$
\begin{aligned}
& H_{k} \widehat{d z}^{k}+A_{I g}^{k} \widehat{\lambda}^{k}+\nabla_{z} f_{r_{k}}\left(x^{k}, y^{k}\right)=0, \\
& S_{I^{g}}^{k}\left(A_{I^{g}}^{k}\right)^{\mathrm{T}} \widehat{d}^{k}+G_{I g}^{k} \widehat{\lambda}^{k}=0 .
\end{aligned}
$$


Since $\left\{r_{k}\right\}$ tends to infinity, and $\lambda^{k}=\widehat{\lambda}^{k-1}-r_{k-1} \tilde{e}, k \geq 1$, such that $\left\{\left\|\widehat{\lambda}^{k}\right\|_{\infty}\right\}$ tends to infinity as well, we have

$$
\beta_{k}=\max \left\{\left\|\widehat{\lambda}_{F, I}^{k}\right\|_{\infty},\left\|\hat{\lambda}_{\Psi_{\varepsilon}, I}^{k}\right\|_{\infty},\left\|\hat{\lambda}_{g, I}^{k}\right\|_{\infty}, 1\right\} \stackrel{K}{\longrightarrow} \infty .
$$

Define

$$
\bar{\lambda}_{F, I^{\mathcal{C}}}^{k}=\beta_{k}^{-1} \widehat{\lambda}_{F, I^{\mathcal{C}}}^{k}, \quad \bar{\lambda}_{\Psi_{\varepsilon}, I^{\mathcal{C}}}^{k}=\beta_{k}^{-1} \widehat{\lambda}_{\Psi_{\varepsilon}, I^{\mathcal{C}}}^{k}, \quad \bar{\lambda}_{g, I^{g}}^{k}=\beta_{k}^{-1} \widehat{\lambda}_{g, I^{g}}^{k},
$$

for $k(\in K)$ large enough, it follows that

$$
\max \left\{\left\|\bar{\lambda}_{F, I^{\mathcal{C}}}^{k}\right\|_{\infty},\left\|\bar{\lambda}_{\Psi_{\varepsilon}, I^{\mathcal{C}}}^{k}\right\|_{\infty},\left\|\bar{\lambda}_{g, I^{g}}^{k}\right\|_{\infty}\right\}=1
$$

Since the sequence $\left\{z^{k}\right\}_{k \in K}$ is bounded by Assumption 3, there exists an infinite index set $K^{\prime} \subseteq K, z^{*} \in \mathbb{R}^{n+2 m}$, and $\left(\bar{\lambda}_{F, I}^{*}, \bar{\lambda}_{\Psi_{\varepsilon}, I^{\mathcal{C}}}^{*}, \bar{\lambda}_{g, I^{g}}^{*}\right) \neq 0$, for all $k \in K^{\prime}$, such that

$$
\lim _{k \rightarrow \infty} z^{k}=z^{*}, \quad \lim _{k \rightarrow \infty} \bar{\lambda}_{F, I^{\mathcal{C}}}^{k}=\bar{\lambda}_{F, I^{\mathcal{C}}}^{*}, \quad \lim _{k \rightarrow \infty} \bar{\lambda}_{\Psi_{\varepsilon}, I^{\mathcal{C}}}^{k}=\bar{\lambda}_{\Psi_{\varepsilon}, I^{\mathcal{C}}}^{*}, \quad \lim _{k \rightarrow \infty} \bar{\lambda}_{g, I^{g}}^{k}=\bar{\lambda}_{g, I^{g}}^{*} .
$$

In view of the boundedness of $\left\{z^{k}\right\}$ and the continuity of the functions, it follows that

$$
\left\{\nabla_{z}\left(v^{k}-F\left(x^{k}, y^{k}\right)\right)\right\}, \quad\left\{\nabla_{z} \Psi_{\varepsilon}\left(y^{k}, v^{k}\right)\right\}, \quad\left\{\nabla_{z} g\left(x^{k}, y^{k}\right)\right\},
$$

are bounded. Further, $\left\{S_{I g}^{k}\right\}$ are bounded by construction. Multiplying both sides of (41) by $\beta_{k}^{-1}$, we have

$$
\beta_{k}^{-1} S_{I^{g}}^{k}\left(A_{I^{g}}^{k}\right)^{\mathrm{T}} \widehat{d z}^{k}+\beta_{k}^{-1} G_{I^{g}}^{k} \widehat{\lambda}^{k}=0 .
$$

Letting $k \stackrel{K^{\prime}}{\longrightarrow} \infty$, the condition (i) shows that $\beta_{k}^{-1} G_{I^{g}}^{k} \widehat{\lambda}^{k} \stackrel{K^{\prime}}{\longrightarrow} 0$, implying that $\beta_{k}^{-1} \widehat{\lambda}_{F, i}^{k} \stackrel{K^{\prime}}{\longrightarrow} 0$ for $i \in I^{\mathcal{C}} \backslash I_{0}^{F}\left(z^{*}\right)$. Similarly, $\beta_{k}^{-1} \widehat{\lambda}_{\psi_{\varepsilon}, i}^{k} \stackrel{K^{\prime}}{\longrightarrow} 0$ for $i \in I^{\mathcal{C}} \backslash I_{0}^{\Psi_{\varepsilon}}\left(z^{*}\right)$.

From (10) and (42), we know that $\left\{\frac{r_{k}}{\beta_{k}}\right\}$ converges to $a \geq 0$ as $k \stackrel{K^{\prime}}{\longrightarrow} \infty$, with

$$
\bar{\lambda}_{F, i}^{*}=a, \quad \forall i \in I^{\mathcal{C}} \backslash I_{0}^{F}\left(z^{*}\right) ; \quad \bar{\lambda}_{\Psi_{\varepsilon}, i}^{*}=a, \quad \forall i \in I^{\mathcal{C}} \backslash I_{0}^{\Psi_{\varepsilon}}\left(z^{*}\right)
$$

Then it follows from the condition (ii) and (42) that

$$
\bar{\lambda}_{F, i}^{*} \leq a, \quad \forall i \in I_{0}^{F}\left(z^{*}\right) ; \quad \bar{\lambda}_{\Psi_{\varepsilon}, i}^{*} \leq a, \quad \forall i \in I_{0}^{\Psi_{\varepsilon}}\left(z^{*}\right) ; \quad \bar{\lambda}_{g, i}^{*} \leq 0, \quad \forall i \in I_{0}^{g}\left(z^{*}\right)
$$

And from (41), we obtain

$$
s_{g, i}^{k} \nabla_{z} g_{i}\left(x^{k}, y^{k}\right)^{\mathrm{T}} \widehat{d z}^{k}+g_{i}\left(x^{k}, y^{k}\right) \widehat{\lambda}_{g, i}^{k}=0, \quad i \in I^{g},
$$

furthermore, multiplying both sides of this equation by $\beta_{k}^{-1}$, together with the condition (i) and taking the limit as $k \stackrel{K^{\prime}}{\longrightarrow} \infty$, we obtain

$$
g_{i}\left(x^{*}, y^{*}\right) \widehat{\lambda}_{g, i}^{*}=0, \quad i \in I^{g},
$$

so $\widehat{\lambda}_{g, i}^{*}=0$ for all $i \in I^{g} \backslash I_{0}^{g}\left(z^{*}\right)$. 
In view of the condition (i) and the continuity of the functions, multiplying (40) by $\beta_{k}^{-1}$ and taking the limit as $k \stackrel{K^{\prime}}{\rightarrow} \infty$, we get $\beta_{k}^{-1} A_{I g}^{k} \widehat{\lambda}^{k} \stackrel{K^{\prime}}{\longrightarrow} 0$, i.e.,

$$
\sum_{i \in I^{\mathcal{C}}} \bar{\lambda}_{F, i}^{*} \nabla_{z}\left(v_{i}^{*}-F_{i}\left(x^{*}, y^{*}\right)\right)+\sum_{i \in I^{\mathcal{C}}} \bar{\lambda}_{\Psi_{\varepsilon} i}^{*} \nabla_{z} \psi_{\varepsilon}\left(y_{i}^{*}, v_{i}^{*}\right)+\sum_{i \in I_{0}^{g}\left(z^{*}\right)} \bar{\lambda}_{g, i}^{*} \nabla_{z} g_{i}\left(x^{*}, y^{*}\right)=0 .
$$

Since $\bar{\lambda}_{F, I^{\mathcal{C}}}^{*}, \bar{\lambda}_{\Psi_{\varepsilon}, \mathcal{C}}^{*}$, and $\bar{\lambda}_{g, I^{g}}^{*}$ are not zero, combining with (45) and Assumption 2, it follows that

$$
I_{0}^{F}\left(z^{*}\right) \neq\{1, \ldots, m\}, \quad I_{0}^{\Psi_{\varepsilon}}\left(z^{*}\right) \neq\{1, \ldots, m\},
$$

i.e., $z^{*} \notin X$ and $a>0$.

Based on (43), dividing both sides of (45) by $a$, we obtain

$$
\begin{aligned}
& \sum_{i \in I^{\mathcal{C}} \backslash I_{0}^{F}\left(z^{*}\right)} \nabla_{z}\left(v_{i}^{*}-F_{i}\left(x^{*}, y^{*}\right)\right)+\sum_{i \in I^{\mathcal{C}} \backslash I_{0}^{\Psi_{\varepsilon}}} \nabla_{z} z_{z} \psi_{\varepsilon}\left(y_{i}^{*}, v_{i}^{*}\right) \\
& =-\sum_{i \in I_{0}^{F}\left(z^{*}\right)} \frac{\bar{\lambda}_{F, i}^{*}}{a} \nabla_{z}\left(v_{i}^{*}-F_{i}\left(x^{*}, y^{*}\right)\right)-\sum_{i \in I_{0}^{\Psi_{\varepsilon}}\left(z^{*}\right)} \frac{\bar{\lambda}_{\Psi_{\varepsilon}, i}^{*}}{a} \nabla_{z} \psi_{\varepsilon}\left(y_{i}^{*}, v_{i}^{*}\right) \\
& \quad-\sum_{i \in I_{0}^{g}\left(z^{*}\right)} \frac{\bar{\lambda}_{g, i}^{*}}{a} \nabla_{z} g_{i}\left(x^{*}, y^{*}\right) .
\end{aligned}
$$

Adding both sides of the above equality to

$$
\sum_{i \in I_{0}^{F}\left(z^{*}\right)} \nabla_{z}\left(v_{i}^{*}-F_{i}\left(x^{*}, y^{*}\right)\right)+\sum_{i \in I_{0}^{\Psi_{\varepsilon}}\left(z^{*}\right)} \nabla_{z} \psi_{\varepsilon}\left(y_{i}^{*}, v_{i}^{*}\right)
$$

we obtain

$$
\begin{aligned}
\sum_{i \in I^{\mathcal{C}}} & \nabla_{z}\left(v_{i}^{*}-F_{i}\left(x^{*}, y^{*}\right)\right)+\sum_{i \in I^{\mathcal{C}}} \nabla_{z} \psi_{\varepsilon}\left(y_{i}^{*}, v_{i}^{*}\right) \\
= & \sum_{i \in I_{0}^{F}\left(z^{*}\right)} \lambda_{F, i} \nabla_{z}\left(v_{i}^{*}-F_{i}\left(x^{*}, y^{*}\right)\right)+\sum_{i \in I_{0}^{\Psi_{\varepsilon}}\left(z^{*}\right)} \lambda_{\Psi_{\varepsilon}, i} \nabla_{z} \psi_{\varepsilon}\left(y_{i}^{*}, v_{i}^{*}\right) \\
& +\sum_{i \in I_{0}^{g}\left(z^{*}\right)} \lambda_{g, i} \nabla_{z} g_{i}\left(x^{*}, y^{*}\right)
\end{aligned}
$$

where $\lambda_{F, i}=1-\frac{\bar{\lambda}_{F, i}^{*}}{a}, i \in I_{0}^{F}\left(z^{*}\right) ; \lambda_{\Psi_{\varepsilon}, i}=1-\frac{\bar{\lambda}_{\Psi_{\varepsilon}, i}^{*}}{a}, i \in I_{0}^{\Psi_{\varepsilon}}\left(z^{*}\right) ; \lambda_{g, i}=-\frac{\bar{\lambda}_{g, i}^{*}}{a}, i \in I_{0}^{g}\left(z^{*}\right)$. In view of (44) and $z^{*} \notin X$. This contradicts Assumption 4 , so the penalty parameter $r_{k}$ is updated at most a finite number of times.

Lemma 4 Suppose that Assumptions 1-4 hold, then there exists an integer $k_{0}$, such that $\varepsilon_{k} \equiv \varepsilon_{k_{0}}$ for $k \geq k_{0}$. 
Proof By Step 3(2) and Step 5 of Algorithm A, the sequence $\left\{\varepsilon_{k}\right\}$ is monotonically decreasing and bounded from below, so the sequence $\left\{\varepsilon_{k}\right\}$ is convergent. Let

$$
\begin{aligned}
& K_{1}=\left\{k \in K \mid \varepsilon_{k}>\xi_{k+1}\right\}, \\
& K_{2}=\left\{k \in K \mid \widehat{d z}^{k}=0, \widehat{\lambda}_{g, I_{k}}^{k} \geq 0, I^{\mathcal{C}}\left(y^{k}, v^{k}, \varepsilon_{k}\right) \neq \emptyset\right\},
\end{aligned}
$$

where $K=\{0,1,2, \ldots\}$. Now we turn to prove that $K_{1}$ and $K_{2}$ are finite sets.

By contradiction, if $K_{1}$ is infinite set, then $\lim _{k \rightarrow \infty} \varepsilon_{k}=0$. There exist $i \in I^{\mathcal{C}}$ and $\bar{K}_{1} \subseteq K_{1}$, such that

$$
\xi_{k+1}=\sqrt{\left(y_{i}^{k+1}\right)^{2}+\left(v_{i}^{k+1}\right)^{2}}<\varepsilon_{k}, \quad k \in \bar{K}_{1} \subseteq K_{1} .
$$

Together with the boundedness of $\left\{z^{k+1}\right\}_{\bar{K}_{1}}$, we suppose that $z^{k+1} \stackrel{\bar{K}_{1}}{\longrightarrow} z^{*}$. From (47), we have $\left(y_{i}^{*}\right)^{2}+\left(v_{i}^{*}\right)^{2}=0$, which contradicts Assumption 3. So $K_{1}$ is a finite set. Similarly, we show that $K_{2}$ is finite. Based on the finiteness of $K_{1}$ and $K_{2}$, from the update rule (27) of $\varepsilon_{k}$ and Step 3(2), we can conclude that Lemma 4 holds.

Based on Lemmas 3-4, in the following analysis, we suppose, without loss of generality, that $r_{k} \equiv \widehat{r}, \varepsilon_{k} \equiv \varepsilon$.

\section{Lemma 5 Suppose that Assumptions 1-4 hold. Then}

(1) The coefficient matrix sequence $\left\{M_{I_{k}}\right\}$ is unified invertible, and there exists a positive constant $L$ such that $\left\|\left(M_{I_{k}}\right)^{-1}\right\| \leq L, \forall k \geq 0$.

(2) The sequences $\left\{\left(\widehat{d z}^{k}, \hat{\lambda}^{k}\right)\right\}$ and $\left\{\left(d z^{k}, \lambda^{k}\right)\right\}$ are bounded.

Proof (1) By contradiction, suppose that there exists an infinite subset $K$ such that $\left\|\left(M_{I_{k}}\right)^{-1}\right\| \stackrel{K}{\longrightarrow} \infty$. Since $\left\{z^{k}\right\}$ and $\left\{H_{k}\right\}$ are bounded, and the finite choice of $I_{k}$, without loss of generality, for all $k \in K$, let

$$
I_{k} \equiv I^{\prime}, \quad z^{k} \stackrel{K}{\longrightarrow} z^{*}, \quad H_{k} \stackrel{K}{\longrightarrow} H_{*}, \quad s^{k} \stackrel{K}{\longrightarrow} s^{*} \geq s_{\min } e_{I}>0 .
$$

Denote

$$
\begin{aligned}
A_{I^{\prime}}^{*} & =\left(\begin{array}{ccc}
-\nabla_{x} F\left(x^{*}, y^{*}\right) & 0_{n \times m} & \nabla_{x} g_{I^{\prime}}\left(x^{*}, y^{*}\right) \\
-\nabla_{y} F\left(x^{*}, y^{*}\right) & \nabla_{y} \Psi_{\varepsilon}\left(y^{*}, v^{*}\right) & \nabla_{y} g_{I^{\prime}}\left(x^{*}, y^{*}\right) \\
E_{m} & \nabla_{v} \Psi_{\varepsilon}\left(y^{*}, v^{*}\right) & 0_{m \times m_{g}}
\end{array}\right), \\
G_{I^{\prime}}^{*} & =\operatorname{diag}\left(v^{*}-F\left(x^{*}, y^{*}\right), \Psi_{\varepsilon}\left(y^{*}, v^{*}\right), g_{I^{\prime}}\left(x^{*}, y^{*}\right)\right),
\end{aligned}
$$

then

$$
M_{I_{k}} \stackrel{K}{\longrightarrow} M_{I^{\prime}}^{*}=\left(\begin{array}{cc}
H_{*} & A_{I^{\prime}}^{*} \\
S_{I^{\prime}}^{*}\left(A_{I^{\prime}}^{*}\right)^{\mathrm{T}} & G_{I^{\prime}}^{*}
\end{array}\right),
$$

where the matrix $S_{I^{\prime}}^{*}=\operatorname{diag}\left(s_{I^{\prime}}^{*}\right)$ with $s_{I^{\prime}}^{*}=\left(s_{F, I^{\mathcal{C}}}^{*}, s_{\Psi_{\varepsilon}, I^{\mathcal{C}}}^{*}, s_{g, I^{\prime}}^{*}\right)$. 
Under the Assumptions, it can be shown easily that $M_{I^{\prime}}^{*}$ is nonsingular. So we obtain

$$
\left\|\left(M_{I_{k}}\right)^{-1}\right\| \stackrel{K}{\longrightarrow}\left\|\left(M_{I^{\prime}}^{*}\right)^{-1}\right\|<\infty
$$

which contradicts $\left\|\left(M_{I_{k}}\right)^{-1}\right\| \stackrel{K}{\longrightarrow} \infty$.

(2) In view of SLE1 (12), and Lemma 3 as well as $r_{k} \equiv \widehat{r}$, we see that $\left\{\left(\widehat{d r}^{k}, \widehat{\lambda}^{k}\right)\right\}$ is bounded. By (15)-(22) and the boundedness of $\left\{\left(\widehat{d}^{k}, \widehat{\lambda}^{k}\right)\right\}$ as well as the boundedness of $\left\{s^{k}\right\}$, we know that $\left\{\eta_{I_{k}}^{k}\right\}$ is bounded. Hence, the boundedness of $\left\{\left(d z^{k}, \lambda^{k}\right)\right\}$ follows by SLE2 (21).

Lemma 6 Suppose that Assumptions 1-4 hold, $z^{*}$ is an accumulation point of the infinite sequence $\left\{z^{k}\right\}$ generated by Algorithm $\mathrm{A}$, and $\left\{z^{k}\right\}_{K}$ converges to $z^{*}$. If $\left\{\zeta^{k}\right\}_{K} \rightarrow 0$, then $z^{*}$ is a KKT point of the problem (7), and both $\left\{\widehat{\lambda}^{k}\right\}_{K}$ and $\left\{\lambda^{k}\right\}_{K}$ converge to the unique multiplier vector $\lambda^{*}$ corresponding to $z^{*}$.

Proof Let $\left(\widehat{\lambda}^{*} ; \lambda^{*}\right)$ be any accumulation point of $\left\{\left(\widehat{\lambda}^{k} ; \lambda^{k}\right)\right\}_{K}$. First, we verify that $\left(z^{*}, \widehat{\lambda}^{*}\right)$ is a KKT pair of the problem (7). Taking into account Assumption 3, Lemma 5, and the finite choice of $I_{k}$, there exists an infinite index $K^{\prime} \subseteq K$ such that

$$
\begin{aligned}
& I_{k} \equiv I^{\prime}, \quad\left(\hat{\lambda}^{k} ; \lambda^{k}\right) \stackrel{K^{\prime}}{\rightarrow}\left(\hat{\lambda}^{*} ; \lambda^{*}\right), \quad H_{k} \stackrel{K^{\prime}}{\rightarrow} H_{*}, \\
& \widehat{d z}^{k} \stackrel{K^{\prime}}{\rightarrow} \widehat{d z}^{*}, \quad s^{k} \stackrel{K^{\prime}}{\rightarrow} s^{*} \geq s_{\min } e_{I} .
\end{aligned}
$$

It follows from (33), (28), Assumption 3 and $\left\{\zeta^{k}\right\}_{K} \rightarrow 0$ that $\widehat{d z}^{*}=0$. Further, taking the limit in SLE1 (12) for $k \in K^{\prime}$, one obtains

$$
\nabla_{z} f_{\widehat{r}}\left(x^{*}, y^{*}\right)+A_{I^{\prime}}^{*} \widehat{\lambda}_{I^{\prime}}^{*}=0, \quad G_{I^{\prime}}^{*} \widehat{\lambda}_{I^{\prime}}^{*}=0 .
$$

Next we will prove $\widehat{\lambda}_{I^{\prime}}^{*}=\left(\widehat{\lambda}_{F, I}^{*}, \widehat{\lambda}_{\Psi_{\varepsilon}, I^{\mathcal{C}}}^{*}, \widehat{\lambda}_{g, I^{\prime}}^{*}\right) \geq 0$, and $\widehat{\lambda}^{*}=\left(\widehat{\lambda}_{I^{\prime}}^{*}, 0_{I^{g} \backslash I^{\prime}}\right) \geq 0$. From $\widehat{\lambda}_{F, i}^{*}\left(v_{i}^{*}-\right.$ $\left.F_{i}\left(x^{*}, y^{*}\right)\right)=0$, for $i \in I^{\mathcal{C}} \backslash I_{0}^{F}\left(z^{*}\right)$, we have $\widehat{\lambda}_{F, I^{\mathcal{C}} \backslash I_{0}^{F}\left(z^{*}\right)}^{*}=0$. Similarly,

$$
\widehat{\lambda}_{\Psi_{\varepsilon}, I^{\mathcal{C}} \backslash I_{0}^{\Psi_{\varepsilon}}\left(z^{*}\right)}^{*}=0, \quad \widehat{\lambda}_{g, I^{\prime} \backslash I_{0}^{g}\left(z^{*}\right)}^{*}=0 .
$$

By $(18)$ and $\left(\zeta^{k}, \widehat{d z}^{k}\right) \stackrel{K^{\prime}}{\longrightarrow}(0,0)$, one obtains

$$
\sum_{i \in I^{\mathcal{C}}} \frac{\hat{\lambda}_{F, i}^{k}}{s_{F, i}^{k}} \phi_{F, i}^{k}+\sum_{i \in I^{\mathcal{C}}} \frac{\hat{\lambda}_{\Psi_{\varepsilon}, i}^{k}}{s_{\Psi_{\varepsilon}, i}^{k}} D_{\Psi_{\varepsilon}, i}^{k}+\sum_{i \in I^{\prime}} \frac{\widehat{\lambda}_{g, i}^{k}}{s_{g, i}^{k}} Q_{g, i}^{k} \stackrel{K^{\prime}}{\longrightarrow} 0 .
$$

Furthermore, by (32), we have $\frac{\hat{\lambda}_{F, i}^{k} \phi_{F, i}^{k}}{s_{F, i}^{k}} \geq 0$ for every $i \in I^{\mathcal{C}}$. Combining with (49), we have $\widehat{\lambda}_{F, i}^{k} \phi_{F, i}^{k} \stackrel{K^{\prime}}{\longrightarrow} 0, i \in I^{\mathcal{C}}$. So, in view of (15), one has

$$
\widehat{\lambda}_{F, i}^{*} \min \left\{0,-\left(\max \left\{-\widehat{\lambda}_{F, i}^{*}, 0\right\}\right)^{\nu}-C\left(v_{i}^{*}-F_{i}\left(x^{*}, y^{*}\right)\right)\right\}=0, \quad i \in I^{\mathcal{C}},
$$

which implies that $\widehat{\lambda}_{F, i}^{*} \geq 0$ for $i \in I^{\mathcal{C}} \cap I_{0}^{F}\left(z^{*}\right)$. Similarly, one obtains

$$
\widehat{\lambda}_{\Psi_{\varepsilon}, i}^{*} \geq 0 \quad \text { for } i \in I^{\mathcal{C}} \cap I_{0}^{\Psi_{\varepsilon}}\left(z^{*}\right) ; \quad \widehat{\lambda}_{g, i}^{*} \geq 0 \quad \text { for } i \in I^{\prime} \cap I_{0}^{g}\left(z^{*}\right)
$$

So we can claim that $\widehat{\lambda}_{I^{\prime}}^{*} \geq 0$. Obviously, $\widehat{\lambda}^{*}=\left(\widehat{\lambda}_{I^{\prime}}^{*}, 0_{I^{g} \backslash I^{\prime}}\right) \geq 0$ is immediate. 
Therefore, in view of $z^{*} \in \tilde{X}$ and (50), we see that $\left(z^{*}, \widehat{\lambda}^{*}\right)$ is a KKT pair of the problem (7). Moreover, the above analysis indicates that the sequence $\left\{\widehat{\lambda}^{k}\right\}_{K}$ possesses a unique accumulation point $\widehat{\lambda}^{*}$, so $\lim _{k \in K} \widehat{\lambda}^{k}=\widehat{\lambda}^{*}$.

Last, noticing that $\left(\widehat{d z}^{k}, \widehat{\lambda}^{k}\right) \stackrel{K^{\prime}}{\longrightarrow}\left(0, \widehat{\lambda}^{*}\right) \geq 0,(15)-(17)$ and (22), we have

$$
\phi_{F, I^{\mathcal{C}}}^{k} \stackrel{K^{\prime}}{\longrightarrow} 0, \quad D_{\Psi_{\varepsilon}, I^{\mathcal{C}}}^{k} \stackrel{K^{\prime}}{\longrightarrow} 0, \quad Q_{g, I^{\prime}}^{k} \stackrel{K^{\prime}}{\longrightarrow} 0, \quad \eta_{I^{\prime}}^{k}=\left(\eta_{F, I^{\mathcal{C}}}^{k}, \eta_{\Psi_{\varepsilon}, I^{\mathcal{C}}}^{k}, \eta_{g, I^{\prime}}^{k}\right) \stackrel{K^{\prime}}{\longrightarrow} 0 .
$$

Denote

$$
M_{I_{k}}\left(\begin{array}{c}
d z^{k} \\
\lambda_{I^{\prime}}^{k}
\end{array}\right)=\left(\begin{array}{c}
-\nabla_{z} f_{r}\left(x^{k}, y^{k}\right) \\
\eta_{I^{\prime}}^{k}
\end{array}\right),
$$

so it follows from the above SLE and SLE1 (12) that

$$
M_{I_{k}}\left(\begin{array}{c}
d z^{k}-\widehat{d z}^{k} \\
\lambda_{I^{\prime}}^{k}-\widehat{\lambda}_{I^{\prime}}^{k}
\end{array}\right)=\left(\begin{array}{c}
0 \\
\eta_{I^{\prime}}^{k}
\end{array}\right) \stackrel{K^{\prime}}{\longrightarrow}\left(\begin{array}{l}
0 \\
0
\end{array}\right),
$$

which together with Lemma 5(1) gives

$$
\lambda^{*}=\lim _{k \in K^{\prime}} \lambda^{k}=\lim _{k \in K^{\prime}} \widehat{\lambda}^{k}=\widehat{\lambda}^{*} .
$$

Based on the lemmas above, we now present the global convergence of Algorithm A.

Theorem 1 Suppose that Assumptions 1-4 hold. $z^{*}$ is an accumulation point of $\left\{z^{k}\right\}$ generated by Algorithm A, then $z^{*}$ is a KKT point of the MPCC (1).

Proof A. We first show that $z^{*}$ is a KKT point of the problem (6) (called Conclusion A).

Let $K^{\prime}$ be an infinite index set such that

$$
\begin{aligned}
& I_{k} \equiv I^{\prime}, \quad\left(\widehat{\lambda}^{k} ; \lambda^{k}\right) \stackrel{K^{\prime}}{\longrightarrow}\left(\widehat{\lambda}^{*} ; \lambda^{*}\right), \quad H_{k} \stackrel{K^{\prime}}{\longrightarrow} H_{*}, \\
& \widehat{d z}^{k} \stackrel{K^{\prime}}{\longrightarrow} \widehat{d z}^{*}, \quad s^{k} \stackrel{K^{\prime}}{\longrightarrow} s^{*} \geq s_{\min } e_{I} .
\end{aligned}
$$

Denote

$$
\begin{aligned}
A_{I^{\prime}}^{*} & =\left(\begin{array}{ccc}
-\nabla_{x} F\left(x^{*}, y^{*}\right) & 0 & \nabla_{x} g_{I^{\prime}}\left(x^{*}, y^{*}\right) \\
-\nabla_{y} F\left(x^{*}, y^{*}\right) & \nabla_{y} \Psi_{\varepsilon}\left(y^{*}, v^{*}\right) & \nabla_{y} g_{I^{\prime}}\left(x^{*}, y^{*}\right) \\
E_{m} & \nabla_{\nu} \Psi_{\varepsilon}\left(y^{*}, v^{*}\right) & 0
\end{array}\right), \\
G_{I^{\prime}}^{*} & =\operatorname{diag}\left(v^{*}-F\left(x^{*}, y^{*}\right), \Psi_{\varepsilon}\left(y^{*}, v^{*}\right), g_{I^{\prime}}\left(x^{*}, y^{*}\right)\right), \\
M_{I^{\prime}}^{*} & =\left(\begin{array}{cc}
H_{*} & A_{I^{\prime}}^{*} \\
S_{I^{\prime}}^{*}\left(A_{I^{\prime}}^{*}\right)^{\mathrm{T}} & G_{I^{\prime}}^{*}
\end{array}\right),
\end{aligned}
$$

where the matrix $S_{I^{\prime}}^{*}=\operatorname{diag}\left(s_{I^{\prime}}^{*}\right)$ with $s_{I^{\prime}}^{*}=\left(s_{F, I^{\mathcal{C}}}^{*}, s_{\Psi_{\varepsilon}, I^{\mathcal{C}}}^{*}, s_{g, I^{\prime}}^{*}\right)$.

By contradiction, suppose that $z^{*}$ is not a KKT point of the problem (6). From Lemma 5, without loss of generality, suppose that $\lambda^{k}=\widehat{\lambda}^{k-1}-\widehat{r} \tilde{e} \stackrel{K^{\prime}}{\longrightarrow} \widehat{\lambda}^{\prime}$. Therefore, it follows that 
$\left(z^{*}, \widehat{\lambda}^{\prime}\right)$ is not a KKT pair of the problem (6), which implies $\rho\left(z^{*}, \widehat{\lambda}^{\prime}\right)>0$ and $I_{0}^{g}\left(z^{*}\right) \subseteq I_{k}$, $k \in K^{\prime}$ large enough. The following two cases are discussed.

Case I. $z^{*}$ is a KKT point of the problem (7). Then there exists a multiplier $\widehat{\lambda}^{\prime \prime}=$ $\left(\widehat{\lambda}_{I_{*}}^{\prime \prime}, 0_{I^{g} \backslash I^{\prime}}\right) \geq 0$, such that the KKT condition of the problem (7) is satisfied at $\left(z^{*}, \widehat{\lambda}_{I_{*}}^{\prime \prime}\right)$. For $k \in K^{\prime}$ large enough, we have $I_{0}^{g}\left(z^{*}\right) \subseteq I_{k} \equiv I^{\prime}$. It is easy to see that $\left(0, \widehat{\lambda}_{I_{*}^{\prime \prime}}^{\prime \prime}\right)$ is a solution to the following SLE:

$$
M_{I^{\prime}}^{*}\left(\begin{array}{c}
d z \\
\lambda_{I_{k}}
\end{array}\right)=-\left(\begin{array}{c}
\nabla_{z} f_{\bar{r}}\left(x^{*}, y^{*}\right) \\
0
\end{array}\right) .
$$

Passing to the limit in SLE1 (12), we know that $\left(\widehat{d z}^{*}, \widehat{\lambda}_{I_{*}}^{*}\right)$ is the solution to (53) above. Let $\widehat{\lambda}^{*}=\left(\widehat{\lambda}_{I_{*}}^{*}, 0_{I^{g} \backslash I^{\prime}}\right)$. In view of Lemma 5 (1), taking into account the nonsingularity of the matrix $M_{I^{\prime}}^{*}$, we see that the solution of (53) is unique. Hence, we obtain $\widehat{d z}^{*}=0, \widehat{\lambda}_{I_{*}}^{*}=$ $\widehat{\lambda}_{I_{*}}^{\prime \prime} \geq 0$, which implies $\widehat{\lambda}^{*}=\widehat{\lambda}^{\prime \prime} \geq 0$. The conditions (i) and (ii) in Step 3 (2) are satisfied for $k \in K^{\prime}$ large enough. Moreover, in view of $r_{k} \equiv \widehat{r}<\infty$ for $k$ large enough, Step 3 (2) implies $\widehat{\lambda}_{F, I^{\mathcal{C}}}^{k}>\gamma_{3} e_{I^{\mathcal{C}}}, \widehat{\lambda}_{\Psi_{\varepsilon}, I^{\mathcal{C}}}^{k}>\gamma_{4} e_{I^{\mathcal{C}}}$ for $k \in K^{\prime}$ large enough, which further implies that

$$
\widehat{\lambda}_{F, I^{\mathcal{C}}}^{*} \geq \gamma_{3} e_{I^{\mathcal{C}}}>0, \quad \widehat{\lambda}_{\Psi_{\varepsilon}, I^{\mathcal{C}}}^{*} \geq \gamma_{4} e_{I^{\mathcal{C}}}>0 .
$$

Since the pair $\left(z^{*}, \widehat{\lambda}^{\prime \prime}\right)$ satisfies the complementary slackness, one gets

$$
\begin{aligned}
& \widehat{\lambda}_{F, i}^{\prime \prime}\left(v_{i}^{*}-F_{i}\left(x^{*}, y^{*}\right)\right)=\widehat{\lambda}_{F, i}^{*}\left(v_{i}^{*}-F_{i}\left(x^{*}, y^{*}\right)\right)=0, \quad i \in I^{\mathcal{C}}, \\
& \widehat{\lambda}_{\Psi_{\varepsilon}, i}^{\prime \prime} \psi_{\varepsilon}\left(y_{i}^{*}, v_{i}^{*}\right)=\widehat{\lambda}_{\Psi_{\varepsilon}, i}^{*} \psi_{\varepsilon}\left(y_{i}^{*}, v_{i}^{*}\right)=0, \quad i \in I^{\mathcal{C}} .
\end{aligned}
$$

So $v^{*}-F\left(x^{*}, y^{*}\right)=0, \Psi_{\varepsilon}\left(y^{*}, v^{*}\right)=0$, which together with Proposition 2 shows that $z^{*}$ is a KKT point of the problem (6), which contradicts the assumption that $z^{*}$ is not a KKT point of the problem (6).

Case II. $z^{*}$ is not a KKT point of the problem (7). It follows from Lemma 6 and $\zeta^{k} \leq 0$ that $\zeta^{k} \stackrel{K^{\prime}}{\rightarrow} \bar{\zeta}<0$. By (18), (28) and Assumption 3, we have

$$
\lim _{k \in K^{\prime}}\left(\left\|\widehat{d z}^{k}\right\|^{q}+\left\|\varpi^{k}\right\|\right)>0
$$

so there exist a subset $K^{\prime \prime} \subseteq K^{\prime}$ and a positive constant $\bar{c}$ such that

$$
\zeta^{k} \leq \frac{\bar{\zeta}}{2}<0 ; \quad\left(\left\|\widehat{d z}^{k}\right\|^{q}+\left\|\varpi^{k}\right\|\right) \geq \bar{c}>0, \quad k \in K^{\prime \prime}
$$

In the following we first show that there exists a positive constant $\bar{t}$, such that $t_{k} \geq \bar{t}$ for all $k \in K^{\prime \prime}$.

(i) By (24)-(26), we have

$$
\begin{aligned}
& v_{i}^{*}-F_{i}\left(x^{*}, y^{*}\right)<0, \quad i \notin I_{0}^{F}\left(z^{*}\right) ; \quad \psi_{\varepsilon}\left(y_{i}^{*}, v_{i}^{*}\right)<0, \quad i \notin I_{0}^{\Psi_{\varepsilon}}\left(z^{*}\right) ; \\
& g_{i}\left(x^{*}, y^{*}\right)<0, \quad i \notin I_{0}^{g}\left(z^{*}\right) .
\end{aligned}
$$


Based on the boundedness of $\left\{d z^{k}\right\}_{K^{\prime \prime}}$ and the continuity of functions, for all $k \in K^{\prime \prime}$ large enough and $t>0$ sufficiently small, one gets

$$
\left(v_{i}^{k}+t d v^{k}\right)-F_{i}\left(u^{k}+t d u^{k}\right)<0, \quad \psi_{\varepsilon}\left(w_{i}^{k}+t d w^{k}\right)<0, \quad g_{i}\left(u^{k}+t d u^{k}\right)<0 .
$$

In view of $i \in I_{0}^{F}\left(z^{*}\right)$, we have $v_{i}^{*}-F_{i}\left(u^{*}\right)=0$. Similarly, $\psi_{\varepsilon}\left(w_{i}^{*}\right)=0$ for $i \in I_{0}^{\Psi_{\varepsilon}}\left(z^{*}\right)$, and $g_{i}\left(u^{*}\right)=0$ for $i \in I_{0}^{g}\left(z^{*}\right)$. From (22) and SLE2 (21), we have

$$
S_{I_{k}}\left(A_{I_{k}}\right)^{\mathrm{T}} d z^{k}+G_{I_{k}} \lambda_{I_{k}}^{k}=\eta_{I_{k}}^{k}
$$

that is,

$$
\begin{aligned}
& \nabla_{z}\left(v_{i}^{k}-F_{i}\left(u^{k}\right)\right)^{\mathrm{T}} d z^{k}=\frac{\eta_{F, i}^{k}-\lambda_{F, i}^{k}\left(v_{i}^{k}-F_{i}\left(u^{k}\right)\right)}{s_{F, i}^{k}}, \quad i \in I^{\mathcal{C}}, \\
& \nabla_{z} \psi_{\varepsilon}\left(w_{i}^{k}\right)^{\mathrm{T}} d z^{k}=\frac{\eta_{\Psi_{\varepsilon}, i}^{k}-\lambda_{\Psi_{\varepsilon}, i}^{k} \psi_{\varepsilon}\left(w_{i}^{k}\right)}{s_{\Psi_{\varepsilon}, i}^{k}}, \quad i \in I^{\mathcal{C}}, \\
& \nabla_{z} g_{i}\left(u^{k}\right)^{\mathrm{T}} d z^{k}=\frac{\eta_{g, i}^{k}-\lambda_{g, i}^{k}\left(w_{i}^{k}\right)}{s_{g, i}^{k}}, \quad i \in I_{k} .
\end{aligned}
$$

By Taylor's expansion and $\left\|\widehat{d z}^{k}\right\| \geq 0$, for $t>0$ sufficient small, we get

$$
\begin{aligned}
& \left(v_{i}^{k}+t d v^{k}\right)-F_{i}\left(u^{k}+t d u^{k}\right) \\
& \quad=\left(v_{i}^{k}-F_{i}\left(u^{k}\right)\right)+t \nabla_{z}\left(v_{i}^{k}-F_{i}\left(u^{k}\right)\right)^{\mathrm{T}} d z^{k}+o(t) \\
& \quad=\left(v_{i}^{k}-F_{i}\left(u^{k}\right)\right)+t \frac{\eta_{F, i}^{k}-\lambda_{F, i}^{k}\left(v_{i}^{k}-F_{i}\left(u^{k}\right)\right)}{s_{F, i}^{k}}+o(t) \\
& \quad=\left(1-t \frac{\lambda_{F, i}^{k}}{s_{F, i}^{k}}\right)\left(v_{i}^{k}-F_{i}\left(u^{k}\right)\right)+t \frac{1-\varrho_{k}}{s_{F, i}^{k}} \phi_{F, i}^{k}-t \varrho_{k}\left(\left\|\widehat{d z}^{k}\right\|^{q}+\left\|\varpi^{k}\right\|\right) \\
& \leq-t \varrho_{k}\left(\left\|\widehat{d z}^{k}\right\|^{q}+\left\|\varpi^{k}\right\|\right)+o(t) .
\end{aligned}
$$

Furthermore, we consider $\zeta^{k} \leq \frac{\bar{\zeta}}{2}<0$, by (19), (20), and the boundedness of $b_{k}$ by Lemma 5, we see that there exists a constant $\bar{a}>0$ such that $\varrho_{k} \geq \bar{a}>0, k \in K^{\prime \prime}$. For $k \in K^{\prime \prime}$ large enough and $t>0$ small enough, we have

$$
\left(v_{i}^{k}+t d v^{k}\right)-F_{i}\left(u^{k}+t d u^{k}\right) \leq-\bar{a} \bar{c} t+o(t)<0, \quad i \in I_{0}^{F}\left(z^{*}\right) .
$$

Similarly, for $k \in K^{\prime \prime}$ large enough and $t>0$ small enough, we have

$$
\begin{aligned}
& \psi_{\varepsilon}\left(w_{i}^{k}+t d w^{k}\right) \leq-\bar{a} \bar{c} t+o(t)<0, \quad i \in I_{0}^{\psi_{\varepsilon}}\left(z^{*}\right), \\
& g\left(u_{i}^{k}+t d u^{k}\right) \leq-\bar{a} \bar{c} t+o(t)<0, \quad i \in I_{0}^{g}\left(z^{*}\right) .
\end{aligned}
$$


(ii) Consider the inequality (23). By (29) and Taylor expansion, for $k \in K^{\prime \prime}$ large enough and $t>0$ small enough, it follows that

$$
\begin{aligned}
& f_{\hat{r}}\left(z^{k}+t d z^{k}\right)-f_{\widehat{r}}\left(z^{k}\right)-\alpha t \nabla_{z} f_{\hat{r}}\left(x^{k}, y^{k}\right)^{\mathrm{T}} d z^{k} \\
& \quad=(1-\alpha) t \nabla_{z} f_{\hat{r}}\left(x^{k}, y^{k}\right)^{\mathrm{T}} d z^{k}+o(t) \\
& \quad \leq(1-\alpha) t \theta \zeta^{k}+o(t) \leq(1-\alpha) t \theta \frac{\bar{\zeta}}{2}+o(t) \leq 0 .
\end{aligned}
$$

By summarizing the above discussion, we can conclude that the inequalities (23)-(26) hold for $k \in K^{\prime \prime}$ large enough and $t>0$ small enough, that is, $\bar{t}=\inf \left\{t_{k}: k \in K^{\prime \prime}\right\}>0$.

In what follows, based on $t_{k} \geq \bar{t}>0\left(k \in K^{\prime \prime}\right)$ we will deduce a contradiction. In view of the monotone decreasing property of $\left\{f_{\vec{r}}\left(z^{k}\right)\right\}$ and $\lim _{k \in K^{\prime \prime}} f_{\bar{r}}\left(z^{k}\right)=f_{r}\left(z^{*}\right)$, we have $\lim _{k \rightarrow \infty} f_{r}\left(z^{k}\right)=f_{r}\left(z^{*}\right)$. Moreover, from (23) and (29), for $k \in K^{\prime \prime}$ large enough, we have

$$
f_{\widehat{r}}\left(z^{k+1}\right)-f_{\widehat{r}}\left(z^{k}\right) \leq \alpha t_{k} \nabla_{z} f_{\widehat{r}}\left(x^{k}, y^{k}\right)^{\mathrm{T}} d z^{k} \leq \alpha t_{k} \theta \zeta^{k} \leq \alpha \bar{t} \theta \frac{\bar{\zeta}}{2}
$$

Taking the limit $k \stackrel{K^{\prime \prime}}{\rightarrow} \infty$ in the inequality above, we obtain a contradiction.

From the above analysis, we can conclude that $z^{*}$ is a KKT point of the problem (6).

$B$. We now show that $I^{\mathcal{C}}\left(y^{*}, v^{*}, \varepsilon\right)=\emptyset$ (called Conclusion B).

From Lemma 4 and the updating rule of $\varepsilon_{k}$ in Step 5, we see that

$$
\sqrt{\left(y_{i}^{*}\right)^{2}+\left(v_{i}^{*}\right)^{2}} \geq \varepsilon, \quad \forall i \in I^{\mathcal{C}}
$$

which implies that $I^{\mathcal{C}}\left(y^{*}, v^{*}, \varepsilon\right)=\emptyset$.

Based on Conclusions A and B, and combining with Proposition 1, we can conclude that $z^{*}$ is a KKT point of the MPCC (1).

\section{Numerical experiments}

In this section, a preliminary implementation of Algorithm $\mathrm{A}$ is given on a Intel(R) Core(TM) i5-7200U CPU (@2.50 GHz, 2.71 GHz) and RAM (4 GB). A Matlab code (Version R2014a) is written corresponding to this implementation.

In our tests, referring to [21], we initialize $H_{0}=E_{n}$, and for $k=1,2, \ldots$, the approximate Hessian matrix $H_{k}$ is determined by the following equation:

$$
H_{k}:=\nabla_{z z}^{2} L_{r_{k}}\left(z^{k}, s^{k}\right)+h_{k} E_{n+2 m}
$$

where $E_{n+2 m}$ is an $(n+2 m)$ th-order identity matrix, $h_{k}$ is chosen as follows:

$$
h_{k}:= \begin{cases}0, & \text { if } \lambda_{\min }>s_{\max } \\ -\lambda_{\min }+s_{\min }, & \text { if }\left|\lambda_{\min }\right| \leq s_{\max } \\ 2\left|\lambda_{\min }\right|, & \text { otherwise, }\end{cases}
$$


Table 1 The computational results

\begin{tabular}{|c|c|c|c|c|c|c|c|c|}
\hline \multirow[t]{2}{*}{ Problem } & \multirow[t]{2}{*}{$\operatorname{dim}$} & \multirow[t]{2}{*}{$f_{\mathrm{opt}}$} & \multicolumn{2}{|l|}{ Algorithm A } & \multirow{2}{*}{$\frac{\text { filtermpec }}{\text { itr }}$} & \multirow{2}{*}{$\frac{\text { snopt }}{\text { itr }}$} & \multirow{2}{*}{$\frac{\text { loqo }}{\text { itr }}$} & \multirow{2}{*}{$\frac{\text { knitro }}{i t r}$} \\
\hline & & & $\overline{f^{*}}$ & itr & & & & \\
\hline bard3m & 6 & -12.6787 & -12.6787 & 38 & 4 & 10 & 23 & 66 \\
\hline flp2 & 4 & 0 & $6.2848 \mathrm{e}-11$ & 9 & - & - & - & - \\
\hline gauvin & 3 & 20 & 20.0000 & 34 & 7 & 10 & 20 & 29 \\
\hline kth2 & 2 & 0 & $-5.2411 e-06$ & 12 & 2 & 4 & 11 & 7 \\
\hline kth3 & 2 & 0.5 & 0.5000 & 5 & 4 & 6 & 10 & 22 \\
\hline scholtes2 & 3 & 15 & 14.4898 & 15 & 2 & 3 & 11 & 16 \\
\hline scholtes3 & 2 & 0.5 & 0.5000 & 6 & 4 & 0 & 29 & 10 \\
\hline scholtes5 & 3 & 1.0 & 1.0000 & 8 & 1 & 3 & 10 & 8 \\
\hline
\end{tabular}

where $s_{\min }>0$ and $s_{\max }>0$ are sufficiently small and sufficiently large, respectively, $\lambda_{\min }$ is the smallest eigenvalue of the following matrix:

$$
\begin{aligned}
\mathcal{B}_{k}= & \nabla_{z z}^{2} L_{r_{k}}\left(z^{k}, s^{k}\right)-\sum_{i \in I^{\mathcal{C}}} \frac{s_{F, i}^{k}}{v_{i}-F_{i}\left(x^{k}, y^{k}\right)} \nabla_{z}\left(v_{i}-F_{i}\left(x^{k}, y^{k}\right)\right) \nabla_{z}\left(v_{i}-F_{i}\left(x^{k}, y^{k}\right)\right)^{\mathrm{T}} \\
& -\sum_{i \in I^{\mathcal{C}}} \frac{s_{\Psi_{\varepsilon}, i}^{k}}{\psi_{\varepsilon}\left(y_{i}^{k}, v_{i}^{k}\right)} \nabla_{z}\left(\psi_{\varepsilon}\left(y_{i}^{k}, v_{i}^{k}\right)\right) \nabla_{z}\left(\psi_{\varepsilon}\left(y_{i}^{k}, v_{i}^{k}\right)\right)^{\mathrm{T}} \\
& -\sum_{i \in I_{g}} \frac{s_{g, i}^{k}}{g_{i}\left(x^{k}, y^{k}\right)} \nabla_{z} g_{i}\left(x^{k}, y^{k}\right) \nabla_{z} g_{i}\left(x^{k}, y^{k}\right)^{\mathrm{T}} .
\end{aligned}
$$

In the numerical experiments, the parameters are chosen as follows:

$$
\begin{aligned}
& \alpha=0.49, \quad \varepsilon=10^{-5}, \quad \beta=0.5, \quad \theta=0.99, \quad q=3, \quad \tau=2, \\
& r=1, \quad C=100, \quad \vartheta=0.5, \quad v=1, \quad \gamma_{1}=\gamma_{2}=\gamma_{3}=\gamma_{4}=1, \\
& s_{\max }=10^{20}, \quad s_{\min }=10^{-5} .
\end{aligned}
$$

Algorithm A stops if one of the following termination criteria is satisfied:

(1) $\rho\left(z^{k}, \lambda^{k}\right) \leq 10^{-5}$ and $I^{\mathcal{C}}\left(y^{k}, v^{k}, \varepsilon_{k}\right)=\emptyset$.

(2) $\left\|\widehat{d z}^{k}\right\| \leq 10^{-5}$, $\max \left\{-\widehat{\lambda}_{g, i}^{k}, i \in I^{g}\right\}<10^{-5}$ and $I^{\mathcal{C}}\left(y^{k}, v^{k}, \varepsilon_{k}\right)=\emptyset$.

The following test problems are selected from [34]. Algorithm A can find their solutions within a small number of iterations. We compared Algorithm A with four algorithms, i.e., filtermpec, snopt, loqo, and knitro given in [35]. The computational results are given in Table 1. The meaning of some notations in Table 1 are as follows:

Problem: The problem in [34].

dim: the dimensions of the variables $(x, y)$;

itr: the number of iterations;

$f_{\text {opt }}$ : the optimal value given in [34];

$f^{*}$ : the optimal value obtained by Algorithm A.

\section{Conclusions}

In this paper, based on Fischer-Burmeister function and working set techniques, a primal-dual interior point QP-free algorithm for mathematical programs with complementarity constraints is presented. At each iteration, only two reduced linear equations with the same coefficient matrix are solved to yield the search direction. The use of the 
working set decreases the computational cost. Moreover, the uniformly positive definiteness on the Lagrangian Hessian estimate $H_{k}$ is relaxed. Under some mild conditions, the proposed algorithm is globally convergent. The preliminary numerical results show that the proposed algorithm is effective.

\section{Funding}

This research is supported by the National Natural Science Foundation (11561005) of China.

Availability of data and materials

Not applicable.

\section{Competing interests}

The authors declare that they have no competing interests.

\section{Authors' contributions}

The authors completed the paper. The authors read and approved the final manuscript.

\section{Publisher's Note}

Springer Nature remains neutral with regard to jurisdictional claims in published maps and institutional affiliations.

Received: 26 June 2020 Accepted: 14 August 2020 Published online: 27 August 2020

\section{References}

1. Ferris, M.C., Pang, J.S.: Engineering and economic applications of complementarity problems. SIAM Rev. 39, 669-713 (1997)

2. Luo, Z.Q., Pang, J.S., Ralph, D.: Mathematical Programs with Equilibrium Constraints. Cambridge University Press, Cambridge (1996)

3. Kocvara, M., Outrata, J., Zowe, J.: Nonsmooth Approach to Optimization Problems with Equilibrium Constraints: Theory, Applications and Numerical Results. Nonconvex Optimization and Its Applications, vol. 28. Kluwer Academic, Dordrecht (1998)

4. Dempe, S.: Foundations of Bilevel Programming. Nonconvex Optimization and Its Applications, vol. 61. Kluwer Academic, Dordrecht (2002)

5. Fukushima, M.: A successive quadratic programming algorithm with global and superlinear convergence properties. Math. Program. 35, 253-264 (1986)

6. Panier, E.R., Tits, A.L.: A superlinearly convergent feasible method for the solution of inequality constrained optimization problems. SIAM J. Control Optim. 25, 934-950 (1987)

7. Anitescu, M.: A superlinearly convergent sequential quadratically constrained quadratic programming algorithm for degenerate nonlinear programming. SIAM J. Optim. 12, 949-978 (2002)

8. Fukushima, M., Luo, Z.Q., Tseng, P.: A sequential quadratically constrained quadratic programming method for differentiable convex minimization. SIAM J. Optim. 13, 1098-1119 (2003)

9. Jian, J.B.: New sequential quadratically constrained quadratic programming method of feasible directions and its convergence rate. J. Optim. Theory Appl. 129, 109-130 (2006)

10. Solodov, M.V.: Global convergence of an SQP method without boundedness assumptions on any of iterative sequences. Math. Program. 118, 1-12 (2009)

11. Jian, J.B., Tang, C.M., Zheng, H.Y.: Sequential quadratically constrained quadratic programming norm-relaxed algorithm of strongly sub-feasible directions. Eur. J. Oper. Res. 200, 645-657 (2010)

12. Jian, J.B., Liu, Y.: A superlinearly convergent method of quasi-strongly sub-feasible directions with active set identifying for constrained optimization. Nonlinear Anal., Real World Appl. 12, 2717-2729 (2011)

13. Jian, J.B., Hu, Q.J., Tang, C.M.: Superlinearly convergent norm-relaxed SQP method based on active set identification and new line search for constrained minimax problems. J. Optim. Theory Appl. 163, 859-883 (2014)

14. Panier, E.R., Tits, A.L., Herskovits, J.N.: A QP-free, globally convergent, locally superlinearly convergent algorithm for inequality constrained optimization. SIAM J. Control Optim. 26, 788-811 (1988)

15. Gao, Z.Y., He, G.P., Wu, F.: Sequential systems of linear equations algorithm for nonlinear optimization problems with general constraints. J. Optim. Theory Appl. 95, 371-397 (1997)

16. Takahashi, W.: The split feasibility problem in Banach spaces. J. Nonlinear Convex Anal. 15, 1349-1355 (2014)

17. Shang, Y.: A new filter QP-free method for the nonlinear inequality constrained optimization problem. J. Inequal. Appl. 2018, Article ID 278 (2018)

18. Takahahsi, W., Yao, J.C.: The split common fixed point problem for two finite families of nonlinear mappings in Hilbert spaces. J. Nonlinear Convex Anal. 20, 173-195 (2019)

19. Kanzow, C., Qi, H.D.: A QP-free constrained Newton-type method for variational inequality problems. Math. Program. 85, 81-106 (1999)

20. Liu, W.A., Shen, C.G., Zhu, X.J., Pu, D.G.: An infeasible QP-free algorithm without a penalty function or a filter for nonlinear inequality constrained optimization. Optim. Methods Softw. 168, 1-28 (2005)

21. Bakhtiari, S., Tits, A.L.: A simple primal-dual feasible interior-point method for nonlinear programming with monotone descent. Comput. Optim. Appl. 25, 17-38 (2003)

22. Yang, Y.F., Li, D.H., Qi, L.Q.: A feasible sequential linear equation method for inequality constrained optimization. SIAM J. Optim. 13, 1222-1244 (2003) 
23. Chen, L., Wang, Y.L., He, G.P.: A feasible active set QP-free method for nonlinear programming. SIAM J. Optim. 17, 401-429 (2006)

24. Jian, J.B., Pan, H.Q., Tang, C.M., Li, J.L.: A strongly sub-feasible primal-dual quasi interior-point algorithm for nonlinear inequality constrained optimization. Appl. Math. Comput. 266, 560-578 (2015)

25. Jian, J.B., Zeng, H.J., Ma, G.D.: Primal-dual interior point QP-free algorithm for nonlinear constrained optimization. J. Inequal. Appl. 2017, Article ID 239 (2017)

26. Li, J.L., Jian, J.B.: A superlinearly convergent SSLE algorithm for optimization problems with linear complementarity constraints. J. Glob. Optim. 33, 477-510 (2005)

27. Li, J.L., Huang, R.S., Jian, J.B.: A superlinearly convergent QP-free algorithm for mathematical programs with equilibrium constraints. Appl. Math. Comput. 269, 885-903 (2015)

28. Ma, C.F., Liang, G.P.: A new successive approximation damped Newton method for nonlinear complementarity problems. J. Math. Res. Expo. 23, 1-6 (2003)

29. Mayne, D.Q., Polak, E.: Feasible directions algorithms for optimization problems with equality and inequality constraints. Math. Program. 11, 67-80 (1976)

30. Fukushima, M., Luo, Z.Q., Pang, J.S.: A globally convergent sequential quadratic programming algorithm for mathematical programs with linear complementarity constraints. Comput. Optim. Appl. 10, 5-34 (1998)

31. Jiang, H.Y., Ralph, D.: Smooth SQP methods for mathematical programs with nonlinear complementarity constraints. SIAM J. Optim. 10, 779-808 (2000)

32. Facchinei, F., Fischer, A., Kanzow, C.: On the accurate identification of active constraints. SIAM J. Optim. 9, 14-32 (1998)

33. Tits, A.L., Wachter, A., Bakhtiari, S., Urban, T.J., Lawrence, C.T.: A primal-dual interior-point method for nonlinear programming with strong global and local convergence properties. SIAM J. Optim. 14, 173-199 (2003)

34. Leyffer, S.: MacMPEC: AMPL collection of MPECS (2000). http://www.mcs.anl.go/ Leyffer/MacMPEC

35. Fletcher, R., Leyffer, S.: Solving mathematical programs with complementarity constraints as nonlinear programs. Optim. Methods Softw. 19, 15-40 (2004)

\section{Submit your manuscript to a SpringerOpen ${ }^{\circ}$ journal and benefit from:}

- Convenient online submission

- Rigorous peer review

- Open access: articles freely available online

- High visibility within the field

- Retaining the copyright to your article

Submit your next manuscript at $\gg$ springeropen.com 